University of Tennessee Health Science Center

UTHSC Digital Commons

\title{
Effects of Aging and Spectral Shaping on the Sub-cortical (Brainstem) Differentiation of Contrastive Stop Consonants
}

Dania A. Rishiq

University of Tennessee Health Science Center

Follow this and additional works at: https://dc.uthsc.edu/dissertations

Part of the Speech and Hearing Science Commons, and the Speech Pathology and Audiology Commons

\section{Recommended Citation}

Rishiq, Dania A. , "Effects of Aging and Spectral Shaping on the Sub-cortical (Brainstem) Differentiation of Contrastive Stop Consonants" (2013). Theses and Dissertations (ETD). Paper 218. http://dx.doi.org/ 10.21007/etd.cghs.2013.0260.

This Dissertation is brought to you for free and open access by the College of Graduate Health Sciences at UTHSC Digital Commons. It has been accepted for inclusion in Theses and Dissertations (ETD) by an authorized administrator of UTHSC Digital Commons. For more information, please contact jwelch30@uthsc.edu. 


\title{
Effects of Aging and Spectral Shaping on the Sub-cortical (Brainstem) Differentiation of Contrastive Stop Consonants
}

\begin{abstract}
Purpose: The objectives of this dissertation are to: (1) evaluate the influence of aging on the sub-cortical (brainstem) differentiation of voiced stop consonants (i.e. /b-d-g/); (2) determine whether potential aging deficits at the brainstem level influence behavioral identification of the /b-d-g/ stimuli, (3) investigate whether spectral shaping diminishes any aging impairments at the brainstem level; and (4) if so, whether minimizing these deficits improves the behavioral identification of the speech stimuli.

Subjects: Behavioral and electrophysiological responses were collected from 11 older adults (> 50 years old) with near-normal to normal hearing and were compared to those of 16 normal-hearing younger adults (control group).
\end{abstract}

Stimuli and Methods: Speech- evoked auditory brainstem responses (Speech-ABRs) were recorded for three 100-ms long /b-d-g/ consonant-vowel exemplars in unshaped and shaped conditions, for a total of six stimuli. Frequency-dependent spectral-shaping enhanced the second formant (F2) transition relative to the rest of the stimulus, such that it reduced gain for low frequencies; and increased gain for mid and high frequencies, the frequency region of the F2 transition in the /b-d-g/ syllables. Behavioral identification of 15-step perceptual unshaped and shaped / b-d-g/ continua was assessed by generating psychometric functions in order to quantify stimuli perception. Speech ABR peak amplitudes and latencies and stop consonant differentiation scores were measured for 6 stimuli (3 unshaped stimuli and 3 shaped stimuli).

Summary of Findings: Older adults exhibited more robust categorical perception, and subtle sub-cortical deficits when compared to younger adults. Individual data showed fewer expected latency patterns for the /b-d-g/ speech-ABRs in older adults as opposed to younger adults, especially for major peaks. Spectral shaping improved the stop consonant differentiation score for major peaks in older adults, such that it moved older adults in the direction of the younger adults' responses.

Conclusion: Sub-cortical impairments at least those measured in this study do not seem to influence the behavioral differentiation of stop consonants in older adults. On the other hand, cue enhancement by spectral shaping seems to overcome some of the deficits noted at the electrophysiological level.

However, due to a possible ceiling effect, improvements to the originally robust perception of older adults, at the behavioral level were not found.

Significance: Aging seems to reduce the sub-cortical responsiveness to dynamic spectral cues without distorting the spectral coding as evident by the "reparable" age-related changes seen at the electrophysiological level. Cue enhancement appears to increase the neural responsiveness of aged but intact neurons, yielding a better sub-cortical differentiation of stop consonants.

\section{Document Type}

Dissertation

Degree Name

Doctor of Philosophy (PhD)

Program

Speech and Hearing Science 
Research Advisor

Ashley W. Harkrider, Ph.D.

\section{Keywords}

Aging, Second Formant (F2) Transition, Spectral Shaping, Speech-evoked Auditory Brainstem, Response (Speech-ABR), Stop Consonants, Sub-cortical Differentiation

\section{Subject Categories}

Communication Sciences and Disorders | Medicine and Health Sciences | Speech and Hearing Science | Speech Pathology and Audiology 
Effects of Aging and Spectral Shaping on the Sub-cortical (Brainstem)

Differentiation of Contrastive Stop Consonants

\author{
A Dissertation \\ Presented for \\ The Graduate Studies Council \\ The University of Tennessee \\ Health Science Center
}

In Partial Fulfillment

Of the Requirements for the Degree

Doctor of Philosophy

From The University of Tennessee

By

Dania A. Rishiq

May 2013 
Copyright (C) 2013 by Dania A. Rishiq. All rights reserved. 


\section{DEDICATION}

With love and appreciation to my family, friends and teachers. 


\section{ACKNOWLEDGEMENTS}

It is with sincere gratitude that I acknowledge the support and help of my advisor Ashley W. Harkrider who played a major role in writing this dissertation, in my Ph.D. study and research, and in my career. Besides my advisor, I would like to express gratitude to my professor Mark S. Hedrick for his valuable guidance and contribution to stimuli preparation, the set-up and design of the behavioral experiment, and the analysis of perceptual data of this dissertation. Many thanks also go to my committee members, Professors Patrick N. Plyler and Gregory C. Petty for their insightful comments that improved the presentation and contents of this dissertation. 


\begin{abstract}
Purpose: The objectives of this dissertation are to: (1) evaluate the influence of aging on the sub-cortical (brainstem) differentiation of voiced stop consonants (i.e. /b-d$\mathrm{g} /)$; (2) determine whether potential aging deficits at the brainstem level influence behavioral identification of the /b-d-g/ stimuli, (3) investigate whether spectral shaping diminishes any aging impairments at the brainstem level; and (4) if so, whether minimizing these deficits improves the behavioral identification of the speech stimuli.
\end{abstract}

Subjects: Behavioral and electrophysiological responses were collected from 11 older adults ( $>50$ years old) with near-normal to normal hearing and were compared to those of 16 normal-hearing younger adults (control group).

Stimuli and Methods: Speech- evoked auditory brainstem responses (SpeechABRs) were recorded for three 100-ms long /b-d-g/ consonant-vowel exemplars in unshaped and shaped conditions, for a total of six stimuli. Frequency-dependent spectralshaping enhanced the second formant (F2) transition relative to the rest of the stimulus, such that it reduced gain for low frequencies; and increased gain for mid and high frequencies, the frequency region of the F2 transition in the /b-d-g/ syllables. Behavioral identification of 15-step perceptual unshaped and shaped /b-d-g/ continua was assessed by generating psychometric functions in order to quantify stimuli perception. Speech ABR peak amplitudes and latencies and stop consonant differentiation scores were measured for 6 stimuli ( 3 unshaped stimuli and 3 shaped stimuli).

Summary of Findings: Older adults exhibited more robust categorical perception, and subtle sub-cortical deficits when compared to younger adults. Individual data showed fewer expected latency patterns for the /b-d-g/ speech-ABRs in older adults as opposed to younger adults, especially for major peaks. Spectral shaping improved the stop consonant differentiation score for major peaks in older adults, such that it moved older adults in the direction of the younger adults' responses.

Conclusion: Sub-cortical impairments at least those measured in this study do not seem to influence the behavioral differentiation of stop consonants in older adults. On the other hand, cue enhancement by spectral shaping seems to overcome some of the deficits noted at the electrophysiological level. However, due to a possible ceiling effect, improvements to the originally robust perception of older adults, at the behavioral level were not found.

Significance: Aging seems to reduce the sub-cortical responsiveness to dynamic spectral cues without distorting the spectral coding as evident by the "reparable" agerelated changes seen at the electrophysiological level. Cue enhancement appears to increase the neural responsiveness of aged but intact neurons, yielding a better subcortical differentiation of stop consonants. 


\section{TABLE OF CONTENTS}

CHAPTER 1. INTRODUCTION ....................................................................................

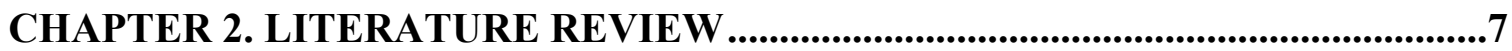

CHAPTER 3. MATERIALS AND METHODS.............................................................13

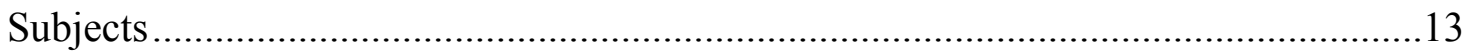

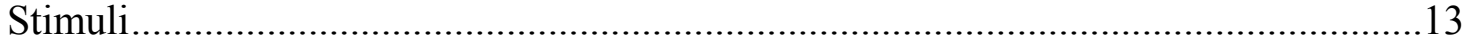

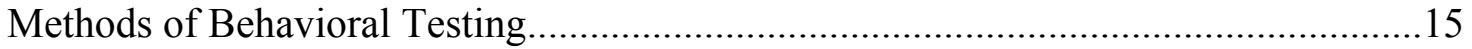

Methods of Electrophysiological Testing .................................................................

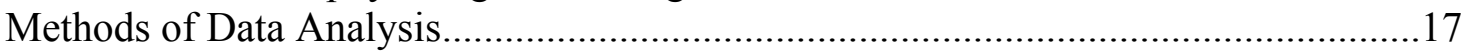

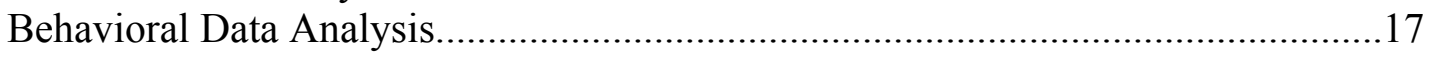

Electrophysiological Data Analysis .................................................................17

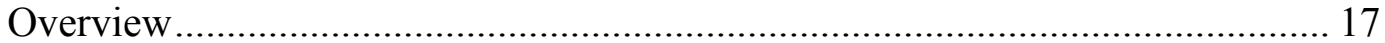

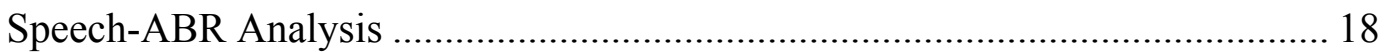

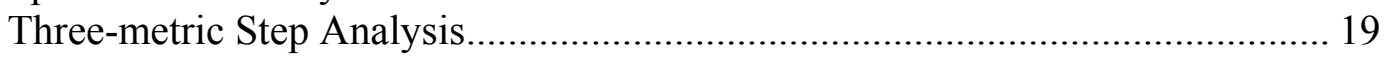

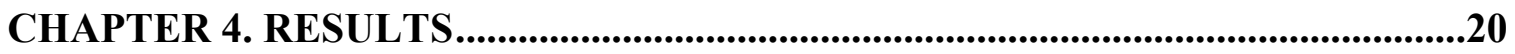

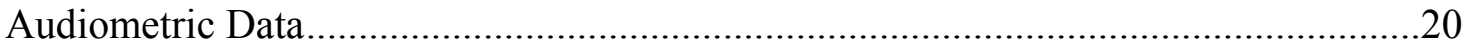

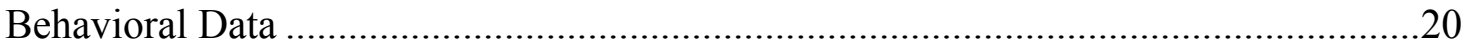

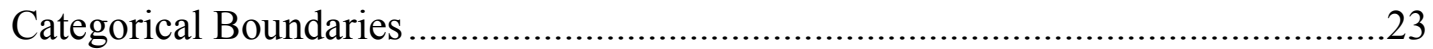

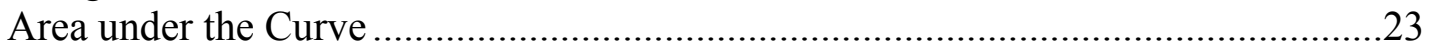

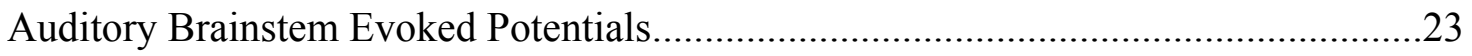

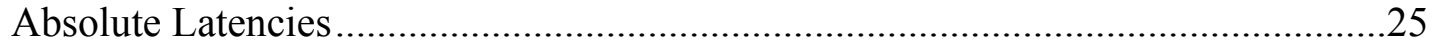

Stop Consonant Differentiation Score ……………..............................................

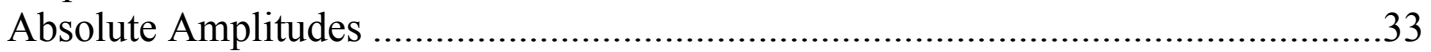

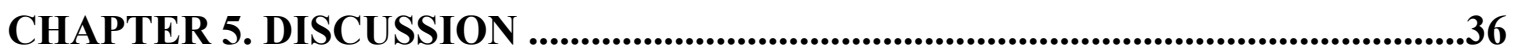

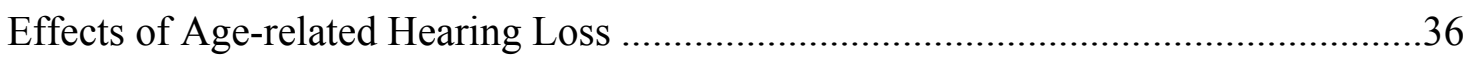

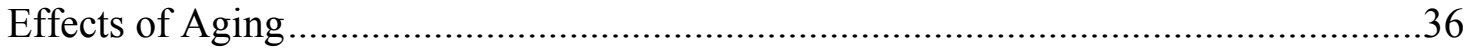

Effects of Spectral Shaping...............................................................................

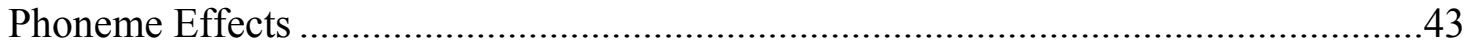

Summary

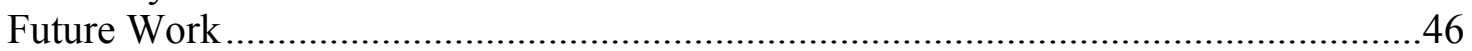

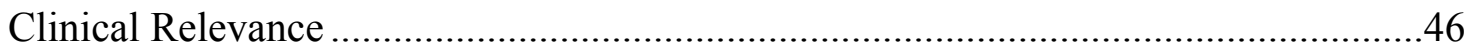

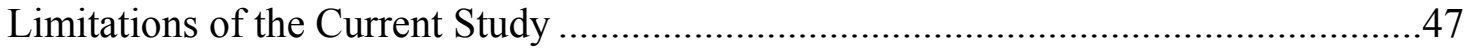

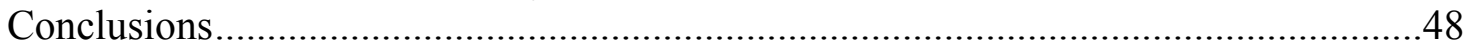

LIST OF REFERENCES ..................................................................................49

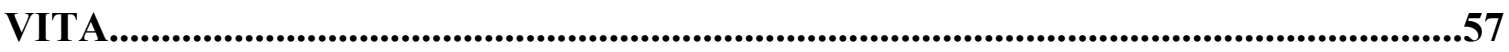




\section{LIST OF TABLES}

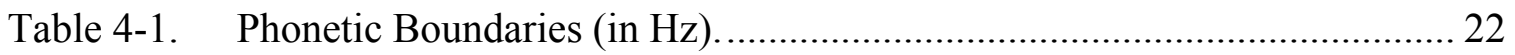

Table 4-2. Area under the Curve..................................................................... 22

Table 4-3. Means and Standard Deviations for All of the Speech-ABR Peaks. .......... 28

Table 4-4. Average Differentiation Scores and Standard Deviations........................ 31

Table 4-5. Correlation Matrix for Behavioral and Electrophysiological Phoneme

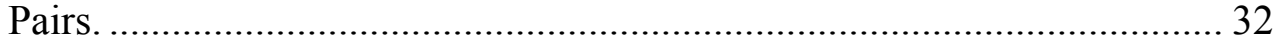

Table 4-6. Significant Results of Paired T-tests for Amplitude Differences among Phoneme Pairs. .................................................................................. 35

Table 4-7. Significant Results of Paired T-tests for Amplitude Differences among Phoneme Pairs in Younger Adults........................................................... 35

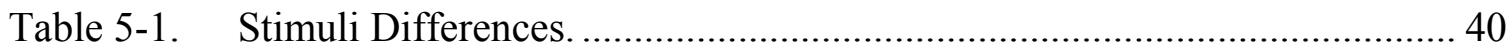




\section{LIST OF FIGURES}

Figure 3-1. Average Audiometric Thresholds for the Test Ear.................................. 14

Figure 4-1. Grand Average Psychometric Functions. .............................................. 21

Figure 4-2. Average Waveforms for Unshaped Phonemes in Controls....................... 24

Figure 4-3. Grand Average Waveforms for Young and Older Adults to Unshaped and Shaped Phonemes. .................................................................. 26

Figure 4-4. Grand Average Waveforms Evoked by the Three Phonemes Are Shown Superimposed for Each Group. .......................................................... 27

Figure 4-5. Average Latency across All Major and Minor Peaks to Unshaped and Shaped Phonemes for Each Individual Subject......................................... 30 


\section{LIST OF ABBREVIATIONS}

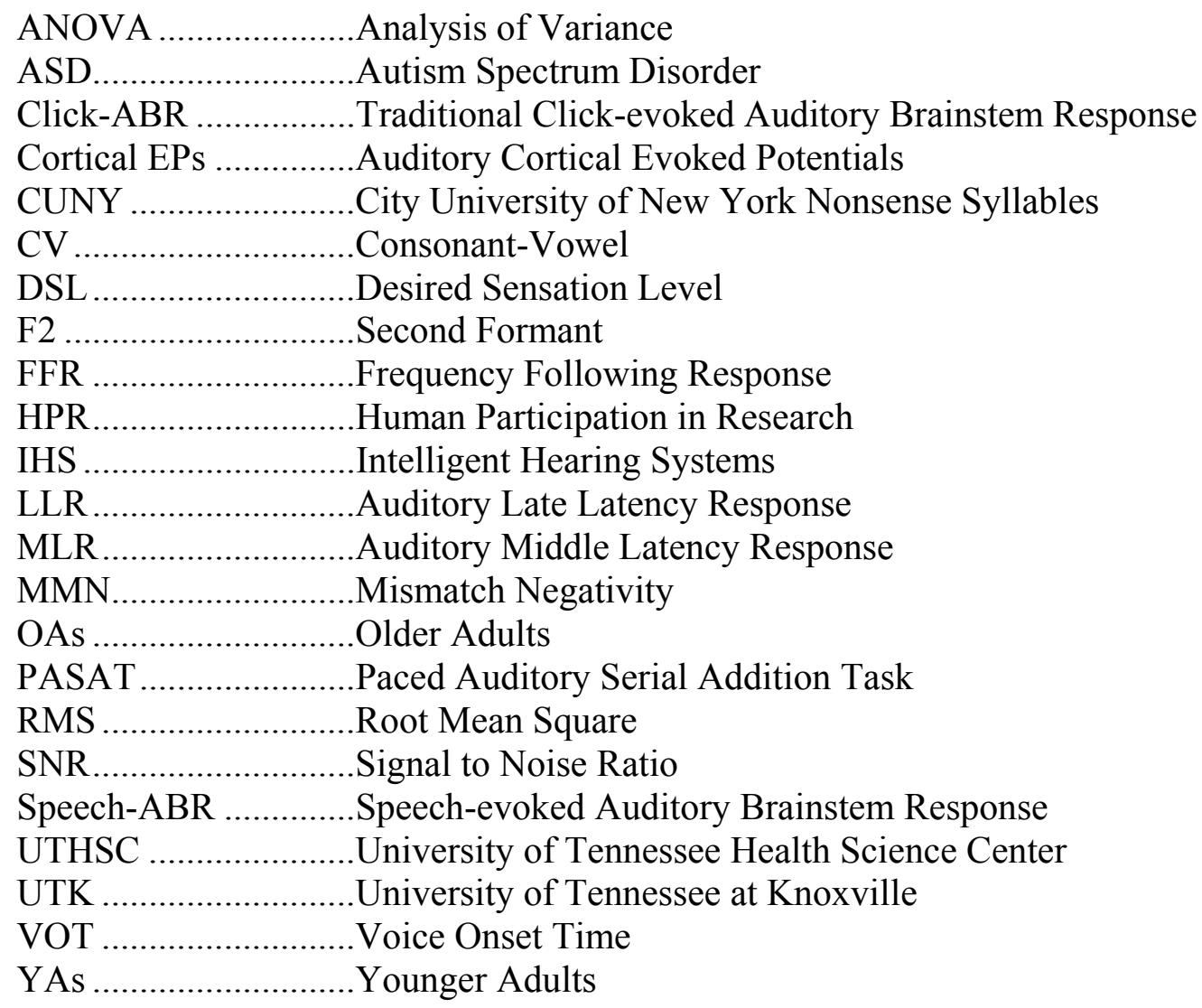




\section{CHAPTER 1. INTRODUCTION}

Older adults even with normal hearing sensitivity often report difficulty discriminating speech sounds (Tremblay, Piskosz \& Souza 2002). Many psychophysical tests, such as silent gap detection, duration discrimination, temporal masking, and temporal order perception reveal impairments in the temporal processing capacities of older adults (Gordon-Salant \& Fitzgibbons 1993; Fitzgibbons \& Gordon-Salant 1996; Lister, Maxfield, Pitt \& Gonzalez 2011). Due to such impairments, the time-varying spectral cues in speech might not be fully captured by the aging auditory system. Hence, the detection of fast temporal changes in the formant transitions that distinguish consonants might be compromised in older adults. Specifically, aging might disrupt the encoding of second formant (F2) transitions in older adults with normal hearing, an imperative speech cue for the identification of place of articulation in consonants (Harkrider, Plyler \& Hedrick 2005).

The effects of age on stop-consonant perception have been investigated behaviorally and electrophysiologically in younger and older adults with and without hearing loss (e.g. Harkrider et al. 2005; Harkrider, Plyler \& Hedrick 2006). Interestingly, investigators found that stop consonant identification performance of older normalhearing adults is similar to that of their hearing-impaired counterparts. On the other hand, identification of stop consonants was poorer in older versus younger adults, heedless of hearing sensitivity. Consistently, the study of Dorman, Marton and Hannley (1985) also explored the effects of age on consonant identification performance, and found that older adults with hearing loss perform similarly to normal-hearing older adults when identifying phonemes on a $/ \mathrm{b}-\mathrm{d}-\mathrm{g} /$ continuum. In this aforementioned study, both groups of older adults, with and without hearing loss, performed poorly when compared to their younger counterparts. Also, the study by Strouse, Ashmead, Ohde and Grantham (1998) reported difficulties discriminating voice onset time (VOT) contrasts of 10 milliseconds in older adults with and without hearing loss versus younger hearing adults.

The effects of aging on the cortical P300 were investigated in several studies in the literature using tonal signals as well as speech signals. For example, $1000 \mathrm{~Hz}$ and $2000 \mathrm{~Hz}$ tone bursts were presented $90 \%$ and $10 \%$ of the time as standard and deviant stimuli, respectively in Picton, Stuss, Champagne and Nelson's (1984) study to collect P300 responses in elderly subjects with normal hearing. Also, the consonant-vowel (CV) syllables [be], [d $\varepsilon]$, and $[\mathrm{p} \varepsilon]$, in addition to simple tonal signals, were used in a study by Wall, Fox, Moenter and Dalebout (1991) to evoke P300 responses in elderly subjects with normal hearing. For both types of stimuli, the latency of P300 was found to be prolonged in older adults with normal hearing versus their younger counterparts. Wall et al. (1991) even reported longer P300 latencies for the CV syllables, compared to the P300 latencies evoked by their tonal signals, at a rate of approximately $1.3 \mathrm{~ms}$ per year.

Furthermore, differences in cortical neural responses, evoked by speech signals, have been reported, such that older adults with normal hearing have prolonged P2 latencies and larger N1 amplitudes versus normal-hearing young adults as demonstrated 
by the Harkrider et al. (2005) study. Moreover, a study by Tremblay, Piskosz and Souza (2003) used a 7-step continuum of stop consonants ranging from /ba/ to /pa/ (for a total of 7 tokens), with increments of 10 milliseconds in VOT for each step. These researchers found prolonged N1 latencies, in response to increased VOT increments, and delayed P2 latencies, in response to all stimuli with all VOT increments, in older listeners with and without hearing loss when compared to their younger counterparts. Investigators concluded that delayed neural synchronous firing (Tremblay et al. 2003), increased refractory periods of neurons, and/ or weaker central inhibition (Harkrider et al. 2005) might be causing these differences in cortical potentials between younger and older listeners with normal hearing. Harkrider et al. (2005) further speculated that aging and aging-related hearing loss distinctly affect neurons, such that aging might reduce the neural responsiveness of neurons whereas hearing loss distorts the conveyed auditory information by destroying neurons. This suggests the possibility that repairing or compensating for the negative effects aging has on neurons may occur by enhancing transmitted information (Harkrider et al. 2005).

The overall amplification of speech seems enticing in overcoming the phoneme categorization limitations in older adults, however, the studies of (Harkrider et al. 2005, 2006; Plyler \& Hedrick, 2002) found that increasing the overall presentation level of contrastive speech syllables does not reduce the differences in categorization abilities between older adults with (Harkrider et al. 2006; Plyler \& Hedrick, 2002) and without (Harkrider et al. 2005) hearing loss when compared to younger hearing adults. The behavioral testing of older adults, with and without normal hearing, using stop consonant stimuli introduced at a spectrally-unshaped (frequency-independent) level of presentation yielded no improvement in their utilization of $\mathrm{F} 2$ formant transitions and hence no improvement in their identification of stop consonants (e.g. Harkrider et al. 2005, 2006). Increasing the level of the speech stimulus is thought to compensate for the reduced neural synchrony and enhance the responsiveness of the neurons to the speech signal (Harkrider et al. 2005). However, high presentation levels might have caused the upward spread of masking, rendering speech understanding difficult (Harkrider et al. 2005).

Consequently, such age-related impairment, which cannot be overcome by increasing the stimulus amplitude, introduces additional limitations onto the benefits of amplification and complicates hearing aid fitting in older adults with hearing loss.

On the other hand, frequency-dependent amplification or the spectral-shaping of the stimuli by enhancing the F2 transition cue relative to other parts of the stimulus, yielded similar phoneme boundary shifts and hence similar performance on the identification of stop consonants between normal-hearing older and younger listeners (Harkrider et al. 2005). Specifically, using the spectrally-shaped stimuli in Harkrider et al. (2005), normal-hearing older adults had /d/ categories similar to their younger counterparts. Spectral-shaping that enhances only the level of F2 transition seems to improve the identification of stop consonants by presumably minimizing the effects of upward spread of masking (Harkrider et al. 2005). Moreover, spectral-shaping altered the neural response patterns to the F2 transition in normal-hearing older adults studied in Harkrider et al. (2005) investigation. Their normal-hearing older adults had prolonged cortical P2 components for the unshaped CV stimuli, but once shaping was introduced, 
their P2 components showed similar latencies to younger adults. Specifically, the spectrally-shaped /b/ and /d/ CVs yielded neural responses in normal-hearing older adults similar to the responses evoked by the unshaped /b/ and /d/ CVs in younger adults.

In Harkrider et al. (2005), investigators used obligatory cortical evoked potentials (i.e. N1 and P2) as the electrophysiological measure to evaluate aging and spectral shaping effects on the neural representation of stop consonants in their normal-hearing older adult population. However, in this current investigation, the speech-evoked brainstem response (speech-ABR) was employed to assess the impacts of aging and spectral-shaping on the neural differentiation of stop consonants at the sub-cortical level in normal-hearing older adults. Among the various reasons behind choosing the speechABR for this current study is that the neural coding of auditory signals is of high fidelity at the brainstem level. Remarkably, the speech-evoked brainstem frequency following responses to speech signals can be played back and still be recognized as speech (Galbraith, Arbagey, Branski, Comerci, \& Rector 1995). Such precision of neural transcription presents the speech-ABR as a method of choice to visualize the minute differences in the neural representation of contrastive stop consonants.

Furthermore, the traditional auditory brainstem response (ABR) is often regarded as a highly synchronous response when compared to auditory middle- and late- latency responses (MLR and LLR). The synchronicity of neuronal firing at the level of the brainstem, including the auditory nerve, cochlear nuclei, lateral lemnisci, and inferior colliculi (Møller \& Jannetta, 1985) forms the general physiologic basis that underlies the ABR (Hall Ed. 2007). In order to generate robust and intact ABR waveforms, neurons should fire synchronously. However, in older adults, it is presumed that the neural system becomes more susceptible to neural desynchronization with age. Therefore, the ABR may capture these degenerative age-related alterations in synchronicity that might take place at the sub-cortical level and may cause declines in speech understanding (Vander Werff \& Burns 2011) in older adults. The speech-ABR may be particularly sensitive to this possible neural desynchronization at the sub-cortical level, due to the gradual onsets of speech signals that have prolonged durations and longer rise times (Song, Banai, Russo \& Kraus 2006). Additionally, in contrast to clicks, speech is an intricate signal that has environmental pertinence (Russo, Nicol, Trommer, Zecker, \& Kraus 2009) and that might introduce additional challenges for a compromised auditory neural system. Moreover, evaluating the integrity of speech processing at the level of the brainstem in normal-hearing older adults is imperative due to its possible contributions to upstream cortical processing (Song et al. 2006).

Another reason underlying the use of speech-ABR in this study is that the neural representation of speech cues using endogenous cortical evoked potentials such as P300 can be affected by attention (Singhal, Doerfling \& Fowler 2002) and memory (Potter, Pickles, Roberts, \& Rugg 1992) capacities of the tested individuals. In an attempt to minimize memory and cognitive interference with study results, Harkrider et al. (2005) used the obligatory cortical evoked potentials (N1 and P2) to evaluate the effects of aging on the perception of speech spectral cues. However, despite the fact that subjects can be unaware of the evoking signals in order to obtain conventional auditory late responses, 
the effects of selective attention on N1 are still under debate (Katz Ed. 2002). The amplitudes of $\mathrm{N} 1$ and $\mathrm{P} 2$ can be affected by attention in a distinctive manner (Hall ed. 2007). Hillyard, Hink, Schwent and Picton (1973) suggests that N1 amplitude can be increased by selective attention, whereas Näätänen (1992) thinks that it is enhanced by the superimposition of a "processing negativity" on the N1 component. The study by Näätänen and Picton (1987) combines both of the latter two views.

On the other hand, many studies reported minimal effects, if none, of attention on the ABR (e.g. Picton \& Hillyard 1974). Neither sleep (e.g. Osterharnmel, Shallop, \& Terkildsen 1985) nor anesthesia (e.g. Samra, Lilly, Rush, \& Kirsh 1984) has substantial effects on the outcome of an ABR evaluation. Therefore, using the ABR that is not affected by attention and wakefulness might be more advantageous for the accurate detection of age differences in the perception of time-varying spectral cues. Its utilization might minimize the influence of attention and memory on these potential differences.

It is worth noting, however, that the traditional click-evoked ABR (click-ABR) results reveal controversial reports regarding the differences between younger and older adults. In spite of the several articles that reported prolonged click-ABR latencies in older adults (e.g. Oku \& Hasegewa 1997); several others reported minute or no differences at all (Anias, Lima \& Kos 2004). Nevertheless, when compared to click-ABRs, the speechABR might impart additional diagnostic information by using speech syllables that have different acoustical structure and contain less high frequency components (Song et al. 2006), where hearing sensitivity differences between young and aged listeners would most likely occur. Aged listeners commonly endure reduced hearing sensitivity for highfrequency sounds (Wingfield, Tun \& Mcoy 2005) which can compromise the evaluation of aging impacts on the click-ABR, a response that primarily depends on intact highfrequency hearing acuity. Hence utilizing speech-ABR when evaluating age effects on the differentiation of contrastive stop consonants might minimize the common and debilitating effects of high-frequency hearing loss on such evaluation in older adults (Pichora-Fuller \& Souza 2003). Moreover, the processing of the intricate structures of speech signals such as formant formants may necessitate different and separate processing mechanisms than that for clicks (Song et al. 2006). For example, Russo et al. (2009) investigated speech- and click-ABRs in subjects with autism spectrum disorder (ASD) and found that despite click-ABRs being within the normal limits, the ASDsubjects demonstrated pervading inadequacy in transcribing speech in quiet and background noise as was evident in abnormal speech-ABR results. It also has been found that children with learning disabilities exhibit abnormal encoding of speech at the level of the brainstem via the speech-ABR (e.g. King, Cunningham, Nicol, Warrier, \& Kraus 2002).

One of the other reasons to use the speech-ABR is that it may use contrastive voiced stop consonants to evoke responses, phonemes that older adults often find difficulty discriminating in real life. Therefore, it may capture the possible sub-cortical deficits in the differentiation processing of these speech sounds in older adults. As described before, the speech- ABR is highly sensitive to the temporal features of sound and is likely to transcribe with much faithfulness many of the acoustical distinctive 
properties of speech (Johnson, Nicol \& Kraus 2005; Banai \& Kraus 2008); hence it is presumed to reflect phonemic contrasts (Hornickel, Skoe, Nicol, Zecker \& Kraus 2009). Due to their differences in place of articulation, the three speech syllables with contrastive characteristics, /ba/, /da/, and /ga/, are expected to produce minimally different speech- ABRs in terms of time (not morphology-wise). The contrastive syllable with the highest second formant is expected to evoke the earliest brainstem response, which is in this case the /ga/, followed by the /da/ then the /ba/. These minor shifts in peak latency are assumed to correspond for the neural representation of frequencies above the phase locking capabilities of the brainstem (Hornickel et al. 2009).

In older adults with possible decreased neural responsiveness, speech-ABRs are expected to degenerate with age. The Vander Werff and Burns (2011) study took the first look at the neural representation of speech signals at the level of the brainstem in older adults. The speech-ABRs obtained in the aforementioned study were evoked by a $40-\mathrm{ms}$ synthesized speech syllable, /da/, the most frequently investigated syllable in brainstem literature. When compared to younger adults, older adults with normal hearing showed amplitude and timing differences at the onset and offset parts of the speech-ABR, respectively. The "neural" onset and offset portions of the speech-ABR are thought to represent the onset burst and the offset of the 40-ms duration of the evoking /da/ stimulus. Such findings support the notion that the neural representation of time-varying acoustic cues might be affected by aging at the brainstem level. Vander Werff and Burns (2011) concluded that this impaired temporal coding found in older adults seems to be attributed to the decrement in neural synchrony caused by aging (e.g. Frisina \& Frisina 1997; Harkrider et al. 2005; Harkrider, Plyler \& Hedrick 2009).

One objective of this current investigation was to study the effects of aging on the neural representation of contrastive voiced stop consonant stimuli at the brainstem level. To do so, speech-ABRs were collected from near normal-hearing older adults and normal-hearing younger adults to three $100-\mathrm{ms}$ speech syllables, $/ \mathrm{ba} /, / \mathrm{da} / \mathrm{and} / \mathrm{ga} /$ that vary in place of articulation. The sub-cortical neural representation of the critical feature, F2 transition, was expected to vary in a predictable manner among responses evoked by these three stimuli (due to different places of articulation) as seen in the Johnson, Nicol, Zecker, Bradlow, Skoe and Kraus (2008) and Hornickel et al. (2009) studies. Specifically, it was predicted that the latency distinctions between the /ba/ vs. /da/, /ba/ vs. /ga/ and /da/ vs. /ga/ syllable pairs will be minimal in older adults compared to younger adults (e.g. Hornickel et al. 2009). Therefore, the results of the 3-step metric analysis used to quantify latency relationships for "unshaped" stop consonant ABRs described in Hornickel et al. (2009) and below will be lower in older adults. It is worth noting that Hornickel et al. (2009) and Johnson et al. (2008) based their observations and conclusions on pediatric data (8-13 year old children). This current evaluation of brainstem differentiation of contrastive stop consonants may allow us to understand how a very specific feature of the speech signal is sub-cortically encoded, which is the F2 formant transition, in adults. Results may provide information on whether the neural encoding of the important acoustic cue, F2 transition, is disrupted at the brainstem level in near normal-hearing older adults when compared to normal-hearing younger adults. 
In addition to the electrophysiologic evaluation of brainstem differentiation of stop consonant stimuli (i.e. /ba/, /da/, and /ga/), the behavioral identification patterns of the same stimuli were obtained in near normal-hearing aged adults and compared to that of normal-hearing younger adults. Psychometric functions were generated for each syllable to examine the differences in phonemic categories between near normal-hearing older listeners and normal-hearing younger listeners. It was predicted that the phonemic categorization abilities might be affected by aging. The behavioral results were then compared to electrophysiological data. It was expected that potential speech-ABR abnormalities might accompany possible impairments in categorization abilities in near normal-hearing aged adults when compared to normal-hearing younger adults (e.g. Harkrider et al. 2005, 2006). Both speech-ABR and the behavioral measures of phonemic categorization provide an opportunity to understand the central brain mediation that underlies the identification and discrimination of the place of articulation feature. Hence, another purpose of this study was to determine whether the possibly disrupted phonemic processing, if any exists, in near normal-hearing older adults due to aging (as might be exhibited by possible abnormal behavioral identification patterns of stop consonants) originates from sub-cortical levels (as might be revealed by potential abnormal speech-ABR results).

A second objective of this study was to investigate whether the spectral-shaping of contrastive voiced stop consonant stimuli (similar to those used in Harkrider et al. 2005 , 2006) by enhancing the F2 transition reduced potential differences in sub-cortical neural representations and behavioral identification of such stimuli between near normalhearing older adults and normal-hearing younger adults. It was expected that potential differences in behavioral identification patterns and speech ABRs between the older versus younger adults would be minimized by introducing spectral-shaping to the stimuli. 


\section{CHAPTER 2. LITERATURE REVIEW}

Speech is a compound and dynamic signal that is abundant in temporal and spectral features. In normal listeners, the central auditory system is capable of extracting and resolving many of these salient features. Certain essential features are important for speech perception and are often used to describe speech signals (Johnson 1997b), such as the fundamental frequency, the harmonic and the formant structures (Abrams \& Kraus 2009). The specific roles of these attributes in speech perception have perplexed investigators over the past two decades (Abrams \& Kraus 2009).

Consonant perception is different than vowel perception and tends to be more complicated. The steady state formants define vowels whereas the fast changing formant transitions define consonants and consonants rely on vowels in their perception (Schnupp, Nelken, \& King 2011). Furthermore, consonant perception is often described as categorical while vowel perception is described as gradual (Schnupp et al. 2011). The duration of formant transitions, friction, and voice onset time are rapidly-presented speech cues or features that result in categorical perception, such that they can cause a sudden shift in the perception of a certain phonetic category into another after crossing a certain phonetic boundary along a speech continuum (Elangovan \& Stuart 2011).

Various studies (e.g. Sharma \& Dorman 1999) examined the categorical perception of contrastive phonemes in different populations using auditory cortical evoked potentials (cortical EPs) to probe the neural correlates of speech processing due to their high temporal resolution (Elangovan \& Stuart 2011). Cortical EPs may be used to study the differences in the central auditory processes that are employed in the discrimination of the articulatory features presented within speech syllables imperative for speech perception. For example, mismatch negativity (MMN) was used to investigate this categorization mechanism in adults (Dehaene-Lambertz 1997; Näätänen, Lehtokoski, Lennest, Cheour, Huotilainen, \& Livonen et al. 1997). Investigators suggested that categorical perception occurs early in time in terms of processing as evident by MMN, which is a pre-attentive (early) component of event related-potentials (Herholz, Lappe \& Pantev 2009). They also concluded that this mechanism involves forming a neural code in sensory memory for phonemic features that is different from that of acoustic features.

A study by Korczak and Stapells (2010) investigated the effects of place of articulation of stop consonants among other articulatory features such as vowel-space contrast, and voiced/voiceless distinctions on cortical EPs (N1, MMN, N2b and P3b) in young normal-hearing adults. They used /bi/ vs. /bu/ (for evaluating vowel-space contrast), /ba/ vs. /da/ (for evaluating place of articulation), and /da/ vs. /ta/ (for evaluating voiced/voiceless distinctions) speech stimuli. All of the latencies and amplitudes of cortical EPs used in their study to evaluate place of articulation were essentially slightly earlier and slightly larger, respectively, for /da/ versus /ba/ stimuli.

Several studies (e.g. Tremblay et al. 2003) reported impaired cortical EP patterns elicited by phonemic contrasts in older adults with and without normal hearing when 
compared to younger adults. The structural and physiological alternations that occur in the central auditory system and accompany advancing age (Willott, Parham, \& Hunter1991) affect the temporal and spectral encoding of speech (Tremblay \& Ross 2007). Categorical perception of many phonemes declines with age such that older adults with (e.g. Dorman et al. 1985) or without normal hearing (e.g. Ginzel, Brahe Pedersen, Spliid \& Andersen 1982) show dislocation in category boundaries when compared to younger adults.

It has been thought that response latency, spectral coding, and neural phaselocking (i.e. Frequency Following Response- FFR) of the cortical coding of stop consonants can be used to extract and analyze timing, formants, and pitch information respectively (Johnson et al. 2008). In parallel, the speech-ABR can use the same analytic modes in exploring the representation of the acoustic cues in speech sounds at the brainstem level (Johnson et al. 2008).

The speech-ABR seems to originate from neurons in the inferior colliculus at the rostral brainstem level (Johnson et al. 2008). It highly relies on the firing synchronicity of these neurons to preserve and conduct the temporal and spectral characteristics of speech sounds (Johnson et al. 2008). It has been suggested that distinct neural processes separately encode the major acoustic characteristics of speech sounds (Kraus \& Nicol 2005), such that the acoustic properties of pitch, formants and segment-level timing can be presented by different speech-ABR components (Johnson et al. 2008). Nevertheless, the exact neural processes behind the representation of these major acoustic characteristics of speech that are imperative for speech perception are yet under speculation (Johnson et al. 2008).

In the case of the $/ \mathrm{ba} /, / \mathrm{da} /$, and /ga/ syllables that were used in this study, what mainly distinguishes these syllables from each other is the F2 formant transition (Hornickel et al. 2009). However, the rostral brainstem cannot phase-lock to such frequency ranges (Wang \& Sachs 1994). Hence, response latency, as a timing measure, would be the most likely choice to evaluate the differences in the neural representations of such contrastive consonants (Johnson et al. 2008). The syllable with the highest frequency would be expected to evoke the earliest response due to the tonotopic representation of the brainstem (Johnson et al. 2008). Similarly, it also has been suggested by Agung, Purdy, McMahon and Newall (2006) and Jacobson, Lombardi, Gibbens, Ahmad and Newman (1992) that, according to their frequency-related latency and amplitude results, the obligatory cortical evoked potentials (N1 and P2) follow the tonotopic distribution of the auditory cortex (as cited in Korczak and Stapells 2010). This unique case where the only difference between syllables is the place of articulation, or the filter characteristics of the vocal tract, will help us trace the neural conduction of formant formants shaped by the vocal tract filtering with better precision at the sub-cortical level and hence understand how the human brainstem encodes this specific acoustic cue in speech sounds (Johnson et al. 2008). The formant transition is one of the essential acoustic cues that underlie the identification of stop consonants (Blumstein, Isaacs \& Mertus 1982); therefore it is of high importance to achieve such aforementioned goal. 
The study by Johnson et al. (2008), recorded electrophysiological differences between the aggregate brainstem responses evoked by the /ba/,/da/, and /ga/ syllables in 22 normal-learning children. Their investigation was first to study how the brainstem encodes the acoustic differences in such contrastive stop consonants. As they hypothesized, the collected responses for each syllable followed this order in their latency: /ga/-ABR, /da/-ABR and then /ba/-ABR. Their results were consistent with that obtained by Korczak and Stapells (2010) for cortical EPs evoked by /ba/ and /da/ syllables in normal young adults. Furthermore, speech-ABR peaks termed as "major" by Johnson et al. (2008) were obtained at the fundamental frequency period and reflected the stimuli glottal pulse, thus conveying pitch information (Johnson et al. 2008). Meanwhile, speech-ABR peaks termed as "minor" exhibited the clearest sub-cortical differences among responses of the three syllables even at the individual subject level. The authors speculated that these minor peaks are the neural manifestation of filter cues conveying formant transition information. The authors further suggested that source and filter cues are encoded by distinct neural mechanisms that can be evaluated discretely using the brainstem response.

To specifically learn which of the acoustic cues in the speech syllable that is incorrectly conducted by the brainstem pathway in normal-hearing older adults may help us precisely identify the degrading effects of aging on the sub-cortical transcription of contrastive speech sounds. For example, a clinician may use minor peaks of the speechABRs, which showed the most robust latency differences in response to the three contrastive speech syllables in Johnson et al. (2008) study, to evaluate normal subcortical transcription of these stimuli at the individual level (Johnson et al. 2008). Subsequently, this work may later help us distinguish between sub-cortical auditory impairments that are caused by aging from that by hearing loss. Harkrider et al. (2006) speculated that age-related neural impairments may have resulted in a decreased neural responsiveness that lead to the cortical EP patterns (using Consonant-Vowel stimuli along a /b-d-g/ continuum) found in older adults with normal hearing and varied from the patterns obtained in younger adults. Meanwhile, neural distortion in encoding speech syllables may have resulted from age-related hearing loss aside from the slow neural reaction (Harkrider et al. 2006). Such information may be used in analyzing responses obtained from older adults with hearing loss in subsequent studies. Specifically, the acoustic feature of focus in this current investigation is associated with place of articulation in stop consonants, a feature that is easily influenced by hearing loss (Boothroyd 1984, 1991). Eventually, this practical information may play a future clinical role in programming hearing aids and/or cochlear implants with better precision and may provide tailored amplification services to older adults with hearing loss that meet their individual difficulties. It may also provide an objective measure for evaluating the outcome and benefits of hearing aids and cochlear implants. Furthermore, the information obtained on speech-ABR differentiation of contrastive stop consonants may predict the speech perception capabilities of difficult to test populations such children with auditory neuropathy (Korczak \& Stapells 2010).

Utilization of the speech-ABR in this current investigation explored the relationship between the brainstem encoding of contrastive speech syllables (/ba/, /da/, 
and $/ \mathrm{ga} /$ ) and aging. It was speculated in this study that if older adults with normal hearing exhibit categorical perception that differed from that of young normal-hearing adults using the behavioral stop consonant identification tasks and if, at the same time, differences in the sub-cortical differentiation of contrastive stop consonants measured by speech-ABRs were obtained between older adults with normal hearing and younger adults, then discriminating contrastive stop consonants (or the conveyance of the formant transitions especially the F2 transition) in older adults with normal hearing would appear to be influenced by both the cortical (as evident by impaired cortical EPs in previous studies) and the sub-cortical levels. Similar to the case of children with learning disabilities, stop consonant discrimination will appear to be influenced by the interaction between bottom-up and top-down (i.e. corticofugal) processes (Hornickel et al. 2009) in older adults.

It is possible that impairments at the cortical level in older adults (as evident by impaired cortical EPs in previous studies) may be caused by deficits in auditory processing at the brainstem level, such that distorted information is conveyed to the cortex (Maruthy \& Mannarukrishnaiah 2008). Additionally, cortical structures may undergo compensatory alternations to overcome the effects of these distorted or delayed inputs by the brainstem (Maruthy \& Mannarukrishnaiah 2008); hence, different cortical EPs patterns will be manifested in older adults when compared to their younger counterparts. Vice versa, the cognitive deficits such as short-term memory impairments that accompany aging (Mitchell, Raye, Johnson \& Greene 2006) may result in impaired sub-cortical processing that may lead to a reduced differentiation of contrastive stop consonants in older adults (e.g. Gibbs 2004). Such a combination of ascending perceptual and descending cognitive influences may underlie the phonemic perception in older adults and may dictate their differentiation of contrastive stop consonants.

On the other hand, this current study also speculated that if the sub-cortical encoding of the contrastive stop consonants does not vary between older adults with normal hearing and younger adults (consistent with the null hypothesis) then the discrimination of stop consonants that mainly differ in the place of articulation or the second formant transition will appear to be primarily mediated at the cortical level. In this case, such a finding may indicate dissociation between sub-cortical and cortical contributions toward phonemic processing in older adults with normal hearing, in which the cortical mediation is the separate and main influence on the phonemic perception in this population.

This latter potential finding may be supported or explained by the notion that phonemic cortical processing is usually regarded as speech-specific, whereas sub-cortical regions are thought to contribute to phonemic processing in a non-linguistic context (Darley, Aronson, \& Brown, 1969; Duffy, 1995). It might be that older adults with normal hearing find difficulty in processing speech-specific information at the cortical level due to structural changes in the cortex (Harris, Dubno, Keren, Ahlstrom, \& Eckert 2009). Furthermore, it has been shown that patients with cortical lesions manifest difficulties discriminating and identifying phoneme contrasts that differ in place of articulation (e.g. Blumstein, Baker \& Goodglass 1977a). The study by Ravizza (2003) 
directly compared phonemic perception abilities among 3 subject groups: patients with sub-cortical lesions $(n=10$, average age $=66$ years old $)$, patients with cortical lesions $(n=$ 9 , average age $=67$ years old) and age-matched controls. Interestingly, patients with subcortical lesions were able to identify syllables, presented at a voicing (/ba/ - /pa/) and place of articulation $(/ \mathrm{ba} /-/ \mathrm{da} /)$ continua, similar to normal controls. However, patients with cortical involvement or lesions performed poorly at the same phonemic perception tasks when compared to patients with sub-cortical involvement (Ravizza 2003). These findings were unique given that the results of previous studies assessing the phonemic processing abilities appeared to demonstrate identical performance for both sub-cortical and cortical groups (Ravizza 2003). The use of an identical set of experimental tasks to test different neurological groups with different lesions allows for figuring out which of the neural structures mediate the phonetic processes (Ravizza 2003).

The reported findings by Vander Werff and Burns (2011), in which normalhearing aged listeners exhibited reduced onset amplitudes and delayed offset latency of the speech-ABR, are consistent with a decrement in neural responsiveness (i.e. synchrony) as authors speculated. Both of the reported age-related impairments in their study pertain to the transient portions (onset and offset parts) of the response that are highly affected by synchronicity. However, no other age-related alternations were detected in other parts of the response that convey filter cues for example. Therefore, if similar sub-cortical representations of contrastive stop consonants were found between older adults and younger adults in the current study despite the previously reported impairments in speech-ABR in this population, it is possible that the reduced neural synchrony commonly found in older adults is not adequately affecting the sub-cortical differentiation of the CV stimuli. It might be that the slow neural conduction (at the subcortical level) is affecting only the conveyance of transient aspects but not the utilization of place of articulation cue in the discrimination of stop consonants. Thus once again it is possible that problems related to phonemic processing, such as poor categorization abilities of stop consonants, in aged listeners are primarily caused by impairments at the cortical level and are not sub-cortically mediated.

In an attempt to determine whether the sub-cortical level is involved in the phonemic processing problems found in aged listeners, this current investigation was set to objectively visualize how the brainstem encodes the differentiation of contrastive stop consonants using speech-ABRs in this population. The three phonemes that were used in evoking the brainstem responses in subjects are minimally different on the F2 transition due to their different places of articulation. The utilization of speech-ABRs in this study may allow us to probe how such filter cues are encoded at the sub-cortical level in older adults and whether there are any age-related brainstem disruptions in the representation of these imperative cues in this population. In summary, the main objective of this investigation was to study the effects of aging on the sub-cortical differentiation of contrastive voiced stop consonants and to probe relations between the results of the behavioral identification of these stimuli and their objective sub-cortical differentiation results using their speech-ABRs. By doing so, origins of phonemic processing mediation are explored in near normal to normal-hearing aged listeners in this study. The second main purpose of this investigation was to study whether the frequency-dependent 
spectral-shaping of these contrastive voiced stop consonant stimuli by enhancing the F2 transition reduces the differences, if any, in the sub-cortical differentiation and the behavioral identification of these stimuli between near normal to normal-hearing aged listeners and normal-hearing younger listeners. 


\section{CHAPTER 3. MATERIALS AND METHODS}

\section{Subjects}

Sixteen younger adults (mean $=20.94$ years; range $=18-33$ years; 6 males) and 11 older adults (mean $=58.60$ years; range $=51-72$ years; 4 males) participated in this study. Subjects were recruited from the student and staff population at the University of Tennessee at Knoxville (UTK) and the Knoxville community. In order to recruit older adults, announcements were distributed around the UTK campus and at local churches, restaurants, grocery stores, and coffee bars, with the permission of their respective directors. Human Participation in Research (HPR) system was used to recruit younger adults from the student body enrolling in "General Psychology" courses at the psychology department at UTK.

Participation was on a first come, first serve basis. In return for their participation, older participants were paid $\$ 30$, whereas younger participants received extra 14 credits in their class. This present research study was approved by the Institutional Review Board of the University of Tennessee Health Science Center (UTHSC). Bilateral audiometric thresholds were obtained in a sound-treated booth for the frequencies 250 , $500,1000,2000,3000,4000,6000$, and $8000 \mathrm{~Hz}$. This current study sought to obtain responses from younger and older adults with normal hearing (i.e. pure-tone thresholds $\leq$ $25 \mathrm{~dB}$ HL). However, responses of two older adult subjects with hearing impairment at frequencies above $2 \mathrm{KHz}$ in their right ears were included in the study analyses; one with a mild high frequency hearing loss, and the other with a high-frequency moderately severe hearing loss. Responses of two other older adults with mild hearing loss at lower frequencies (with thresholds no poorer than $35 \mathrm{~dB} \mathrm{HL}$ and $30 \mathrm{~dB} \mathrm{HL}$ at $250 \mathrm{KHz}$ and 500 $\mathrm{KHz}$, respectively) in their right ears were included as well. All younger adult subjects had audiometric thresholds of $25 \mathrm{~dB}$ HL or better at the tested frequencies in both ears. For both groups, younger and older adults, average audiometric thresholds for the tested (i.e. right) ear are illustrated in Figure 3-1.

By subjects' report, all study participants were right-handed and footed and had no history of recurrent ear infections, emotional or behavioral disorders, drug or alcohol dependency, mental illnesses, or any other central nervous system disorder. All older adult subjects obtained passing scores of $\geq 24$ on the Mini Mental Status Examination before they were included in further participation.

\section{Stimuli}

$\mathrm{A} / \mathrm{ba} /-/ \mathrm{da} /-/ \mathrm{ga} /$ perceptual continuum of 15 steps was created via the cascade configuration of Klatt's formant synthesizer (Klatt, 1980) at a sampling rate of $10 \mathrm{KHz}$. Each token of the continuum was $100 \mathrm{~ms}$ in duration. The duration of both the F1 and F2 formant transitions was $40 \mathrm{~ms}$. The first formant began at $150 \mathrm{~Hz}$ and kept increasing until it reached $750 \mathrm{~Hz}$, where the steady state portion starts. The third and fourth 


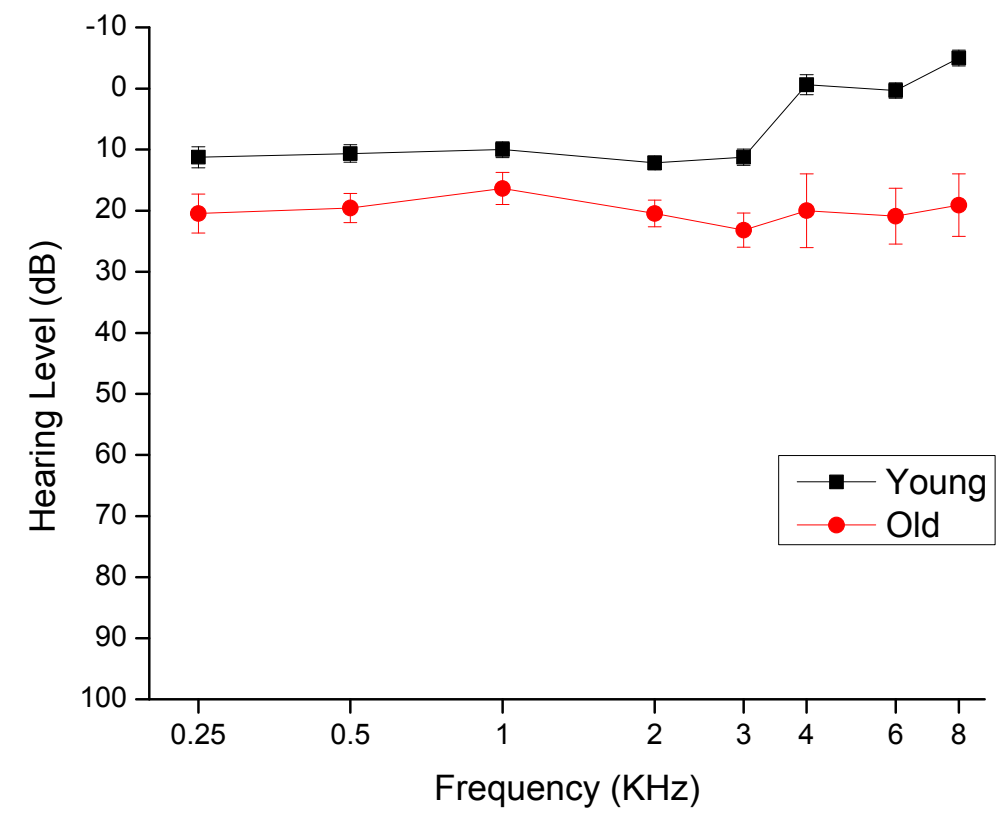

Figure 3-1. Average Audiometric Thresholds for the Test Ear.

Average audiometric thresholds for the right ear of subjects with standard error bars.

Younger and older adults are plotted in filled black squares and red circles, respectively). 
formants remained fixed for the total duration of the stimuli at $2400 \mathrm{~Hz}$ and $3300 \mathrm{~Hz}$, respectively. The starting frequency of the F2 formant ranged from 900 to $2300 \mathrm{~Hz}$ in $100 \mathrm{~Hz}$ steps creating the fifteen tokens of the /ba-da-ga/ continuum. In relation to the first formant, the amplitudes of the $\mathrm{F} 2$ and $\mathrm{F} 3$ formants were $-3 \mathrm{~dB}$ and $-6 \mathrm{~dB}$, respectively.

Cool Edit Pro (Adobe), a signal processing software, was used to spectrally-shape the speech stimuli in order to improve audibility of the F2 formants in relation to the rest of the signal and to minimize the upward spread of masking. Specifically, spectral shaping delivered amplification to speech signals similar to that delivered by the Desired Sensation Level fitting method (DSL v4.1 software, 1996) to conversational speech for a listener with a mild-to-moderate sensorineural hearing loss. This older DSL version was used to facilitate direct comparisons of our obtained results to that of previous studies by Harkrider and colleagues $(2005 ; 2006 ; 2009)$. Spectral shaping reduced gain for low frequencies; and increased gain for mid and high frequencies, which is the frequency region of F2 transitions. Spectral shaping was determined by measuring 1/3 octave root mean square (RMS) levels in a Zwislocki coupler via a sound level meter with slow averaging and C- weighting (Harkrider et al. 2009).

For behavioral testing, the RMS output of the shaped stimuli was equal to that of the unshaped stimuli (i.e. $80 \mathrm{~dB}$ SPL) to ensure that potential behavioral or electrophysiological response differences are due to shaping not because of differences in overall presentation levels (Harkrider et al. 2009). However, more amplification was introduced to the frequencies of F2 formant transition in relation to the rest of the signal in the shaped condition, meanwhile equal amplification was delivered to all frequencies in the unshaped condition. All stimuli were calibrated using a sound level meter with slow averaging and $\mathrm{C}$-weighing.

\section{Methods of Behavioral Testing}

The behavioral testing was administered in the Psychoacoustics Lab in the Department of Audiology and Speech Pathology at the University of Tennessee Health Science Center located in Knoxville, TN. The speech syllables were presented by a signal generation system (Tucker-Davis Technologies, System II) that is interfaced to a microcomputer via an interactive signal generation and control software (CSRE Version 4.5). The stimuli were directed through a digital-to-analog converter, a low pass filter, a digital attenuator, then to a headphone buffer and eventually presented to the right ear through an insert earphone. Subjects were seated in a sound-treated booth viewing a computer screen. They were asked to indicate which of the speech syllables they perceived, by clicking on the appropriate letter (B, D, and $\mathrm{G})$ on display.

Prior to experimental testing, a practice session was administered using six exemplars. Two exemplars (one unshaped and one shaped) were used for each phoneme. The exemplars had 900, 1600 and $2300 \mathrm{~Hz}$ for their F2 onset frequencies. Two independent listeners judged whether these exemplars are considered best examples of 
the $/ \mathrm{ba} /, / \mathrm{da} /$, and $/ \mathrm{ga} /$. In this practice session, each one of the six exemplars was introduced five times for a total of thirty presentations. Thus, the practice session comprised 30 presentations. Once the subject correctly perceived the presented exemplars $50 \%$ of the time (15 out of 30), the experimental testing began. On average one to three practice sessions were introduced before subjects achieved the $50 \%$ criterion (e.g. Harkrider et al. 2005, 2006).

For the experimental testing, 20 blocks, each has 30 token stimuli, were presented for each subject. Thus, a total of 600 responses were collected from each subject. Token stimuli were presented randomly in each trial. The percentage of phoneme identification as a function of onset frequency was expressed in psychometric functions for the shaped and unshaped stimuli. Behavioral testing took approximately 30 minutes.

\section{Methods of Electrophysiological Testing}

Electrophysiological testing was conducted in the Human Auditory Physiology Lab at the Department of Audiology and Speech Pathology of the University of Tennessee Health Science Center in Knoxville, TN. Speech-ABRs were recorded using Intelligent Hearing Systems (IHS) via the SmartEP Advanced Auditory Research Module. The speech-ABRs were acquired using six stop consonant exemplars (one unshaped and one shaped, for each phoneme) selected from the stimuli generated for the /b-d-g/ continuum used in the behavioral testing of this current study. The chosen exemplars represented the best examples of $/ \mathrm{b}-\mathrm{d}-\mathrm{g} /$ phonemes based on the judgment of two independent listeners (Harkrider et al. 2005). The exemplars identically shared all stimuli characteristics except for the transition of their second formant. Exemplars had F2 onset frequencies of $900 \mathrm{~Hz}, 1700 \mathrm{~Hz}$ and $2300 \mathrm{~Hz}$ for the /b/,/d/ and /g/ phonemes, respectively. This current study aimed to minimize the differences among the $/ \mathrm{b}-\mathrm{d}-\mathrm{g} /$ stimuli eliciting the speech-ABRs to facilitate tracing the transformation of acoustic cues to brainwaves. In order to convert stimulus wav files into IHS stimulus format, their sampling rate was up-sampled to $40 \mathrm{KHz}$ and they were uploaded into the conversion utility of SmartEP. After conversion, stimuli were played without any errors. A total of 6 average speech-ABR waveforms [3 phonemes (/ba/, /da/, and /ga/) X 2 shaping condition (unshaped and shaped)] were collected for each subject.

The stop consonant stimuli were presented monaurally to the right ear via an insert earphone at $80 \mathrm{~dB}$ SPL (e.g. Hornickel et al. 2009). The left ear remained unoccluded. The speech-ABRs were collected via a vertical electrode montage where three $\mathrm{Ag}-\mathrm{AgCl}$ cup electrodes were placed on the center of the head (i.e. $\mathrm{Cz}$ : non-inverting/ active), the ipsilateral earlobe (i.e. inverting/ reference) and the forehead (i.e. common ground). The areas of electrode placement were cleansed with a mild scrubbing gel $(\mathrm{Nu}$ Prep). Electrodes were securely placed on the skin with a conductive electrode paste and plugged into the OPTI- amp 8002 transmitter box. Contact impedance was checked and was no more than $5 \mathrm{k} \Omega$ for each electrode. Subjects were comfortably seated in a recliner with the neck at rest in a sound treated booth. They were asked to stay relaxed, 
still and quiet throughout testing. Electrophysiological testing took approximately 2 hours and 15 minutes.

Stimuli were presented in an alternating polarity and at a presentation rate of 4.35 stimuli per second (Johnson et al. 2008). Two blocks of 2000 sweeps were collected for each stimulus resulting in a total of 4000 sweeps per stimulus. The two blocks were averaged/added offline to form the final waveform (of 4000 sweeps) to a given stimulus. Sweeps with artifacts that fell in the range of $\pm 35 \mu \mathrm{V}$ were rejected. A recorded time window (epoch time) of $230 \mathrm{~ms}$ that includes a pre-stimulus interval of $40 \mathrm{~ms}$ and poststimulus interval of $190 \mathrm{~ms}$ were used (Hornickel et al. 2009). In order to track the precision with which these syllables are represented in the ABR, a sufficiently high sampling rate of $75 \mu$ s was used. Data points of 3072 were used. Responses were bandpass filtered from 100 to $3000 \mathrm{~Hz}$ to separate the frequencies of the brainstem (Johnson et al. 2008; Skoe, Nicol, \& Kraus. 2011).

\section{Methods of Data Analysis}

\section{Behavioral Data Analysis}

Per each phoneme and shaping condition, the area under the curve of the psychometric functions (Hedrick, Schulte, \& Jesteadt 1995) was measured. This was done via adding the number of percent responses for each phoneme (/b/, /d/, or $/ \mathrm{g} /)$ collected across the continuum of F2 onset transitions. The sum was divided over the total number of transition steps for the continuum. Additionally, phonetic boundary locations associated with $50 \%$ points were computed for every psychometric function (e.g., Harkrider et al. 2009). The 50\% points were defined by converting the percent unshaped $/ \mathrm{b} /$ scores to $\mathrm{Z}$ scores and using linear least-squares fit to get an estimation of the $50 \%$ point which is the phonemic boundary of this phoneme. Phonemic boundaries were determined using this method for the six stop consonant syllables ( 3 phonemes X 2 shaped/unshaped conditions) and were measured in Hz. These two aforementioned measures, phonemic boundaries and area under the curve, were used to quantify the perception of stop consonants that differ in place of articulation presented in the perceptual continuum (Harkrider et al. 2005).

\section{Electrophysiological Data Analysis}

\section{Overview}

The most studied brainstem response in the literature, and that is most commercially available, is the speech-ABR evoked by a 40-ms /da/. It consists of seven major peaks (V-O): V, A, C, D, E, F, and O, marked consecutively on the response waveform (all are negative peaks except for peak V) (e.g. Johnson et al. 2005; Vander Werff \& Burns 2011). In several other studies (e.g. Johnson et al. 2008; Hornickel et al. 
2009; Skoe et al. 2011) that investigated the sub-cortical differentiation of 170-ms voiced stop consonants, the labeling letters $\mathrm{V}, \mathrm{A}, \mathrm{C}, \mathrm{D}, \mathrm{E}, \mathrm{F}$ and $\mathrm{O}$ were not used. Instead, investigators used a numerical labeling system and recorded 16 peaks ( 6 positive and 10 negative) for the speech-ABRs. They tracked the waveforms in higher precision enabling examination of minute negative peaks that succeeded a major negativity. In these studies, the "minor" peaks were labeled 5, 8,11, and 14, whereas the rest of peaks were termed as "major" peaks. In these studies, the following peaks visually appeared at the following latencies (Note: positive and negative signs used here denote a peak or trough, respectively): waves +1 and -2 (onset peaks) appeared at approximately $9 \mathrm{~ms}$ of the waveform, whereas waves +3 and -4 ; waves +6 and -7 ; waves +9 and -10 ; waves +12 and -13; and waves +15 and -16 (endpoint peaks) consecutively occurred every ten minutes after $23 \mathrm{~ms}$ of the waveform and ended by around $63 \mathrm{~ms}$. Latencies were identified for the first $70 \mathrm{~ms}$ of waveforms because F2 transition- related differences among phonemes were expected to diminish upon reaching the shared steady-state portion at around $50 \mathrm{~ms}$ (i.e. the formant transition duration). Please refer to Johnson et al. (2008) and/or Hornickel et al. (2009) for schematic illustrations of the aforementioned described waves. In these two studies, waves 1 and 2 were termed the onset response since they were presumed to represent the onset of the speech syllable (i.e. the stop burst) whereas waves 15 and 16 were termed endpoint peaks because they were presumed to reflect the end of the formant transition period. Here, it is important to distinguish between endpoint peaks and the negative offset peak observed in other speech-ABR studies that used the 40-ms /da/ (e.g. Vander Werff \& Burns 2011); endpoint peaks mark the beginning of the steady state portion of the signal, whereas the offset peak marks the end of the 40-ms duration of the $/ \mathrm{da} / \mathrm{stimulus}$. In addition, the minor peaks were thought to represent filter cues as reflected by the formant structure, whereas the major peaks were assumed to be largely driven the fundamental frequency of the speech syllable (Hornickel et al. 2008).

\section{Speech-ABR Analysis}

In this current study, the speech-ABR responses for the /ba/, /da/, and /ga/ were analyzed using the Intelligent Hearing Systems (IHS) software via SmartEP program using a method adapted from previous studies (e.g., Johnson et al. 2008; Hornickel et al. 2009; Skoe et al. 2011). Six grand average waveforms [3 phonemes (/ba/, /da/,/ga/) X 2 shaping conditions (unshaped, shaped)] were produced. Peak latencies (i.e. timing measure) and peak amplitudes (i.e. magnitude measure) were identified for 15 speechABR peaks [the onset peaks: 1, (1); the major peaks: 2, 3, 4, 5, (2), (3), (4), (5); the minor peaks: "b", "c", and "d"; and the endpoint peaks: 6 and (6). Peaks were marked manually using the program cursor on each individual waveform following the grand average waveforms as a reference. Peak amplitudes were measured relative to baselines of no stimulation just before presenting the stimuli (Skoe \& Kraus 2010). In this study, latencies were identified for the first $50 \mathrm{~ms}$ of waveforms because F2 transition- related differences among phonemes were expected to lessen upon reaching the shared steadystate portion at around $40 \mathrm{~ms}$ (i.e. the formant transition duration). 


\section{Three-metric Step Analysis}

For each subject, a stop consonant differentiation score was computed for major and minor peaks, per each shaping condition. A metric analysis of three steps described in the Hornickel et al. (2009) study was conducted to generate these differentiation scores. The first step of this analysis starts with pair-wise latency comparisons among the three /b-d-g/ syllable pairs (i.e. three pairs: /ba/ vs. /da/; /ba/ vs. /ga/; and /da/ vs. /ga/) for each peak. It is expected that /ga/ will evoke the earliest response in time, followed by $/ \mathrm{da} /$ then $/ \mathrm{ba} /$. Therefore, when the pair-wise latency comparisons followed this expected pattern, the given peak was given one point, if not; it was given 0 points. Hence, the total points of this initial analysis of the pair-wise comparisons ( 3 pairs) ranged from 0 to 3 for each peak.

In the second step, difference in average latency and standard deviation for every pair (and for each peak) were computed using the responses of the younger adults group. Then, two upper and lower values for latency (for each peak) were set using the difference average latency \pm 0.67 standard deviations, to account for the $50 \%$ cases in a population distributed normally (Hornickel et al. 2009). When a pair-wise comparison for a given peak followed the expected pattern (as in the first step) and the latency difference of that pair exceeded the upper limit value, another point was summed to the initial total score. On the other hand, a point was deduced from that total when the latency difference of that pair fell below the lower limit. Hence, the total points of this second analysis ranged from -3 to 3 for each peak. Combining the points of both steps yielded a total score that ranged from -3 to 6 points, for each peak. In the last third step, all differentiation scores for all major peaks (i.e. 8 peaks) and all differentiation scores of all minor peaks (i.e. 3 peaks) were summed separately for each subject. Thus, the final score ranged from -24 to 48 for major peaks (i.e. 8 peaks), and -9 to 18 for minor peaks (i.e. 3 peaks). A higher differentiation score for an individual subject implies that the speechABRs of the /b-d-g/ stimuli follow the expected latency pattern, which could also indicate a better sub-cortical differentiation of the stop consonant stimuli (Hornickel et al 2009). 


\section{CHAPTER 4. RESULTS}

\section{Audiometric Data}

For each subject, pure-tone thresholds were obtained at 8 frequencies in the tested (right) ear. All younger adult subjects had normal hearing (i.e. audiometric thresholds of $25 \mathrm{~dB}$ HL or better) at all of the tested frequencies. Although this current study was designed to obtain responses from subjects with normal hearing, yet responses of two older adult subjects with hearing impairment above $2 \mathrm{KHz}$ in their right ears were included in the study analyses; one with a mild high-frequency hearing loss, and the other with a high-frequency moderately severe hearing loss. Responses of two other older adults with mild hearing loss at 250 and $500 \mathrm{KHz}$ in their right ears were included as well. Figure 3-1 illustrates grand average audiometric functions for younger and older adults at each frequency.

Per each frequency, audiometric thresholds were statistically evaluated for significant differences between younger and older adults using one-way Analysis of Variance (ANOVA). The observed P-values were adjusted using Bonferroni correction. The statistical analyses yielded the following for each tested frequency: $250 \mathrm{~Hz}[\mathrm{~F}(1,25)$ $=7.472, p=0.088] ; 500 \mathrm{~Hz}[\mathrm{~F}(1,25)=11.615, p=0.061] ; 1000 \mathrm{H}[\mathrm{F}(1,25)=5.716, p=$ $0.200] ; 2000 \mathrm{~Hz}[\mathrm{~F}(1,25)=13.563, p=0.008] ; 3000 \mathrm{~Hz}[\mathrm{~F}(1,25)=18.004, p<0.001]$; $4000 \mathrm{~Hz}[\mathrm{~F}(1,25)=14.928, p=0.001] ; 6000 \mathrm{~Hz}[\mathrm{~F}(1,25)=25.947, p<0.001] ; 8000 \mathrm{~Hz}$ $[\mathrm{F}(1,25)=28.740, p<0.001]$. Group differences in thresholds were not different below $2000 \mathrm{~Hz}$, at $2000 \mathrm{~Hz}$ mean differences between the groups were less than $8.27 \mathrm{~dB}$, and at and above $4000 \mathrm{~Hz}$ mean group differences were less than $21.77 \mathrm{~dB}$.

\section{Behavioral Data}

A total of six psychometric functions [3 phonemes (/ba/,/da/, /ga/) X 2 shaping conditions (unshaped, shaped) were obtained for each subject. Grand average psychometric functions were created for each phoneme and shaping condition for younger and older adults with error bars of one standard error (Figure 4-1).

In order to quantify the perception of stop consonants along the continuum, categorical boundaries and area under the curve measures were computed. Respectively, Tables 4-1 and 4-2 display the means and standard deviations of phonemic boundaries (in $\mathrm{Hz}$ ) and areas under the curve (in average percent of a specific phoneme) for younger and older adults. The effects of age, phoneme, and shaping condition were assessed via analyses of variance (ANOVAs). Two, three-factor ANOVAs with repeated measures were carried out, one on categorical boundaries and one on the arcsine transformed area under the curve measures. The factors were shaping condition (unshaped vs. shaped), phoneme (/ba/ vs. /da/ vs. /ga/), and age (younger vs. older). 

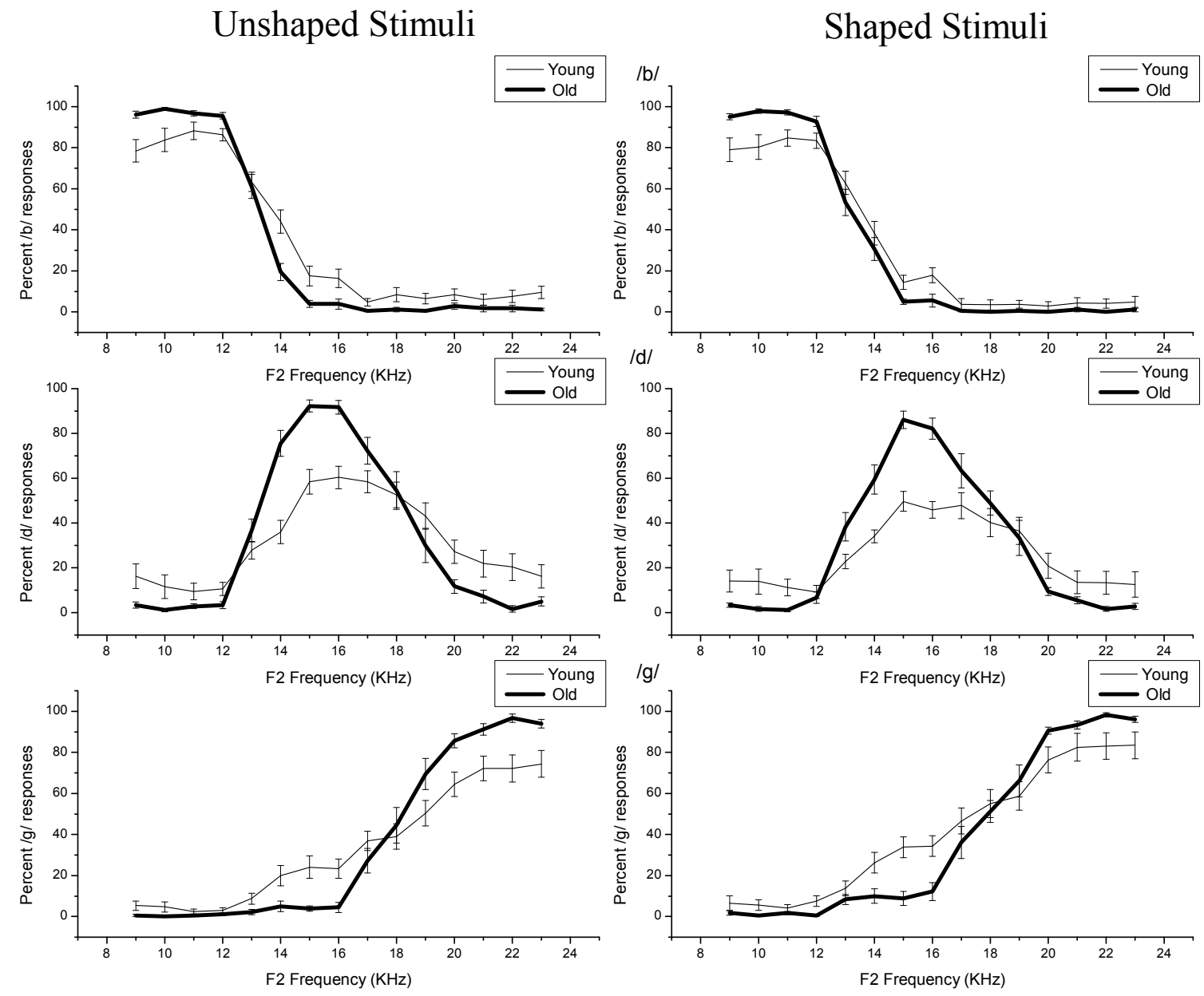

Figure 4-1. Grand Average Psychometric Functions.

Grand average psychometric functions for each phoneme (upper panels for $/ \mathrm{b} /$, mid panels for $/ \mathrm{d} /$, and lower panels for $/ \mathrm{g} /$ ) and shaping condition (left panels for unshaped stimuli, and right panels for shaped stimuli), for younger (YAs) and older adults (OAs) with error bars of one standard error (older adults are plotted in bold lines). 
Table 4-1. Phonetic Boundaries (in Hz).

\begin{tabular}{lrrrrrrrr}
\hline Stimuli & $\mathbf{U S} / \mathbf{b} /$ & $\mathbf{U S} / \mathbf{d}_{\mathbf{b}} /$ & $\mathbf{U S} / \mathbf{d}_{\mathbf{g}} /$ & $\mathbf{U S} / \mathbf{g} /$ & $\mathbf{S} / \mathbf{b} /$ & $\mathbf{S} / \mathbf{d}_{\mathbf{b}} /$ & $\mathbf{S} / \mathbf{d}_{\mathbf{g}} /$ & $\mathbf{S} / \mathbf{g} /$ \\
\hline Younger & & & & & & & & \\
Adults & & & & & & & & \\
Mean & 1325.4 & 1484.0 & 1746.5 & 1845.9 & 1321.1 & 1569.2 & 1660.7 & 1710.7 \\
SD & 147.5 & 137.9 & 218.1 & 175.9 & 183.5 & 181.4 & 256.9 & 169.6 \\
Older & & & & & & & & \\
Adults & & & & & & & & \\
Mean & 1340.1 & 1368.4 & 1796.4 & 1859.2 & 1215.6 & 1391.2 & 1784.5 & 1788.9 \\
SD & 52.7 & 58.4 & 112.3 & 132.5 & 402.1 & 81.5 & 90.9 & 83.7 \\
\hline
\end{tabular}

Table 4-2. Area under the Curve.

\begin{tabular}{lcccccc}
\hline Stimuli & US /b/ & US /d/ & US/g/ & S/b/ & S/d/ & S/g/ \\
\hline Younger Adults & & & & & & \\
$\quad$ Mean & 1.56 & 1.49 & 1.56 & 1.52 & 1.40 & 1.66 \\
$\quad$ SD & 0.11 & 0.14 & 0.13 & 0.12 & 0.13 & 0.11 \\
$\begin{array}{c}\text { Older Adults } \\
\text { Mean }\end{array}$ & 1.54 & 1.54 & 1.57 & 1.53 & 1.50 & 1.61 \\
$\quad$ SD & 0.05 & 0.05 & 0.07 & 0.05 & 0.07 & 0.05 \\
\hline
\end{tabular}


Mauchly's Test of Sphericity was conducted to assess whether the assumption of sphericity was violated for the main effect of the 3-level phoneme factor or for its interaction with the other within-subject factor, shaping condition. In order to correct for the violations of sphericity, if they existed, the $p$-values and adjusted degrees of freedom by the Greenhouse-Geisser correction were reported and used in the determination of the significance of the main effect of phoneme and its interaction with shaping condition for the categorical boundaries and area under the curve variables. P-values $<0.05$ were considered significant. However, P-values were adjusted for multiple comparisons by dividing by 2 , such that $\mathrm{P}$-values $<0.025$ were considered significant.

\section{Categorical Boundaries}

For the categorical boundaries measure, there was no group difference between younger and older adults $[\mathrm{F}(1,25)=0.386, p=0.540]$. Categorical boundaries shifted higher in frequency from $/ \mathrm{ba} /$ to $/ \mathrm{da} /$ to $/ \mathrm{ga} /$, respectively $[\mathrm{F}=(1.024,25.604)=5.750, \mathrm{p}=$ $0.023)$. Also, there was no obtained main effect for the shaping condition $[\mathrm{F}(1,25)$ $=0.027, p=0.872]$, nor any significant interaction.

\section{Area under the Curve}

For the area under the curve variable, a group difference was obtained $[\mathrm{F}(1,24)$ $=11.097, p=0.003]$, such that the area under the curve measure was larger for older adults than for younger adults. A significant main effect for phoneme was reported $[\mathrm{F}(2,23)=$ $6.984, p=0.002]$, indicating that the $/ \mathrm{b} /$ and $/ \mathrm{g} /$ categories were larger than the $/ \mathrm{d} /$ category. A significant shaping by phoneme interaction was also found $[\mathrm{F}(2,23)=$ $10.688, p=0.001]$. To assess the effects of shaping on each particular phoneme, follow up paired t-tests were conducted comparing the area under the curve measure before and after the introduction of shaping for each phoneme. Shaping reduced the area under the curve measure for the $/ \mathrm{b} /[\mathrm{t}(26)=2.155, p=0.041]$ and $/ \mathrm{d} /[\mathrm{t}(26)=2.826, p=0.009]$ categories but increased it for the $/ \mathrm{g} /$ category $[\mathrm{t}(25)=-5.112, p<0.001]$.

\section{Auditory Brainstem Evoked Potentials}

Six individual speech-ABR waveforms measured from the $\mathrm{Cz}$ electrode were collected for each subject [ 3 phonemes (/ba/, /da/, /ga/) X 2 shaping conditions (unshaped, shaped)]. For each phoneme and shaping condition, individual waveforms were averaged together throughout younger adults to create 6 grand average waveforms. In reference to grand average waveforms, the following 15 peaks were marked on each individual waveform for each subject in the present study. The peaks were onset peaks [i.e. expressed in waves 1 and (1)], major peaks [i.e. expressed in waves 2, 3, 4, 5, (2), (3), (4), and (5)], minor Peaks [i.e. expressed in waves a, b and c] and endpoint peaks [expressed in waves 6, (6)]. For a clearer illustration of peaks numeration and classification of this study, please refer to Figure 4-2. 


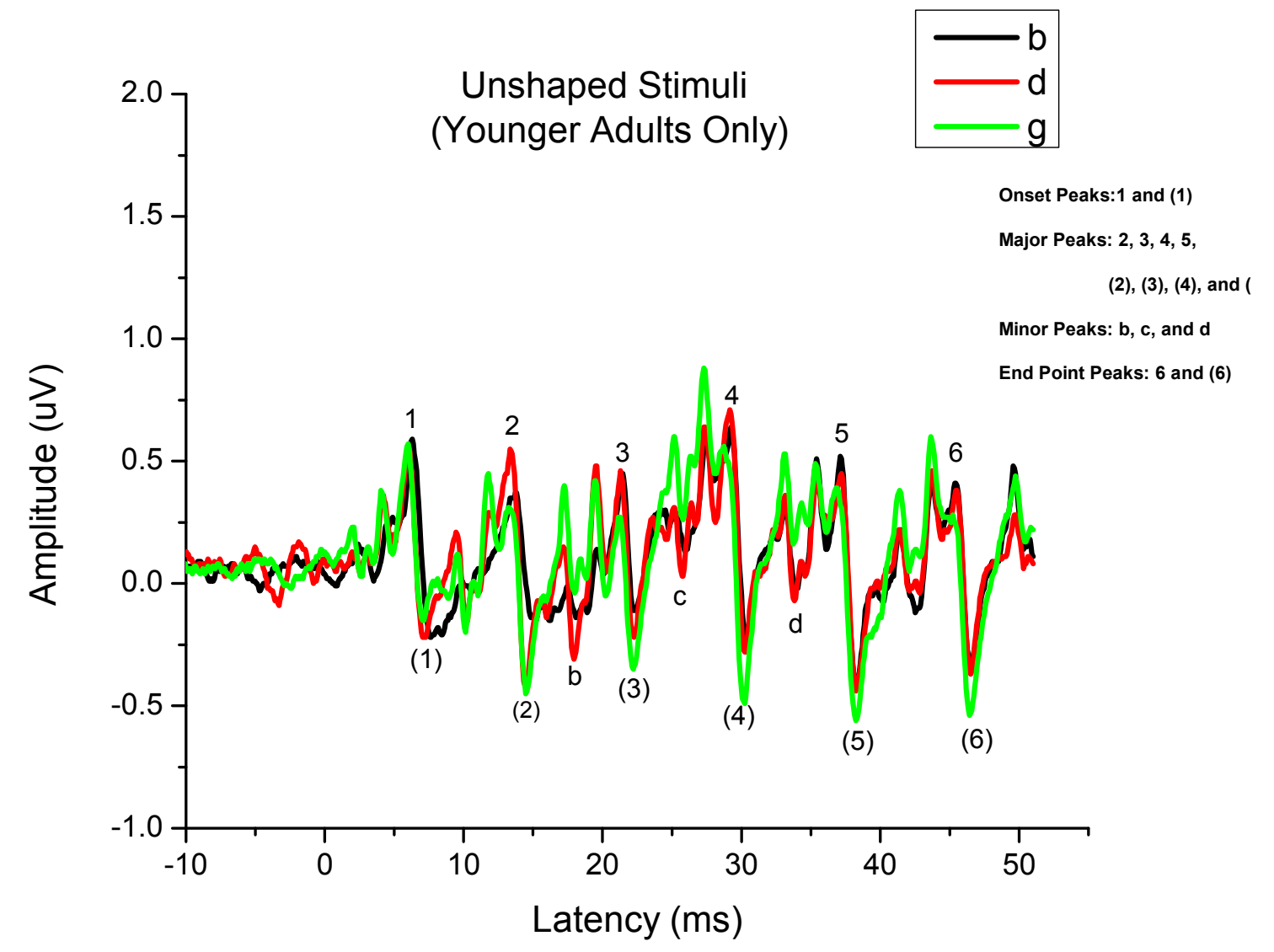

Figure 4-2. Average Waveforms for Unshaped Phonemes in Controls.

Three average waveforms, superimposed on one another, evoked by the three unshaped phonemes, i.e. /b/ (black), /d/ (red), and /g/ (green) stimuli, recorded in younger adult $(\mathrm{n}=16)$ of the present study. Peaks are identified on the waveforms. 
Figure 4-3 illustrates the six grand average waveforms recorded in both younger and older adults (older adults are plotted in bold) and evoked by the unshaped (left panels) and shaped (right panels) stimuli/phonemes $[/ \mathrm{b} /, / \mathrm{d} /, / \mathrm{g} /$ are shown in top, middle, and bottom panels, respectively]. To better visualize phonemic contrasts at the subcortical level, Figure 4-4 shows the three superimposed grand average waveforms evoked by the three phonemes for every group and for both shaping conditions.

\section{Absolute Latencies}

Table 4-3 illustrates the means and standard deviations for all of the speech-ABR peaks evoked by the unshaped and shaped stop consonant stimuli used in this study in our younger $(n=16)$ and older $(n=11)$ adults.

A 3 X 2 X K X 2 repeated measures ANOVA was performed on the latencies of onset peaks, major peaks, minor peaks, and endpoint peaks, separately, where K denotes the number of peaks. The factors were phoneme (repeated measures on 3 levels, /b/ vs. /d/ vs. /g/), shaping condition (repeated measures on 2 levels, shaped vs. unshaped), peaks (repeated measures on K levels: 2 for onset peaks, 8 for major peaks, 3 for minor peaks and 2 for endpoint peaks), and age ( 2 levels, young vs. old).

For the onset peaks, there were no obtained group differences between younger and older adults $[\mathrm{F}(1,24)=3.117, p=0.090]$ nor any significant main effects or interactions. As in onset peaks, not significant was the main effect for age on the endpoint peaks $[\mathrm{F}(1,24)=0.936, p=0.343]$. However, shaping significantly increased the latencies of the endpoint peaks $[\mathrm{F}(1,24)=4.489, p=0.045]$.

As for major peaks, there were no obtained significant group differences between younger and older adults $[\mathrm{F}(1,24)=1.008, p=0.325]$. However, a significant phoneme by shaping interaction was found $[\mathrm{F}(2,23)=3.536, p=0.037]$. To assess the effects of shaping on all major peaks for each particular phoneme, a follow up paired t-tests was conducted comparing latencies of all major peaks (collapsed all over the 8 peaks) before and after the introduction of shaping, for each one of the three phonemes. In overall, shaping reduced the latencies of major peaks for the $/ \mathrm{b} /$ phoneme $[\mathrm{t}(215)=3.484$, $p=0.001]$, in particular.

As for the minor peaks, there were no obtained significant group differences between younger and older adults $[\mathrm{F}(1,24)=0.001, p=0.977]$. However, a significant shaping by peak interaction was obtained $[\mathrm{F}(2,23)=3.796, p=0.029]$. To assess the effects of shaping on each particular minor peak, a paired t-test was conducted comparing the latencies of every minor peak (collapsed all over the 3 phonemes) before and after the introduction of shaping. There were no significant effects for shaping on each one of the $\mathrm{b}[\mathrm{t}(79)=1.353, p=0.180], \mathrm{c}[\mathrm{t}(79)=1.781, p=0.079]$, and $\mathrm{d}$ minor $p$ eaks $[\mathrm{t}(79)=-0.932$, $p=0.354]$. Average latency across all minor and major peaks for each phoneme, for each shaping condition, for each individual subject is illustrated in Figure 4-5. 

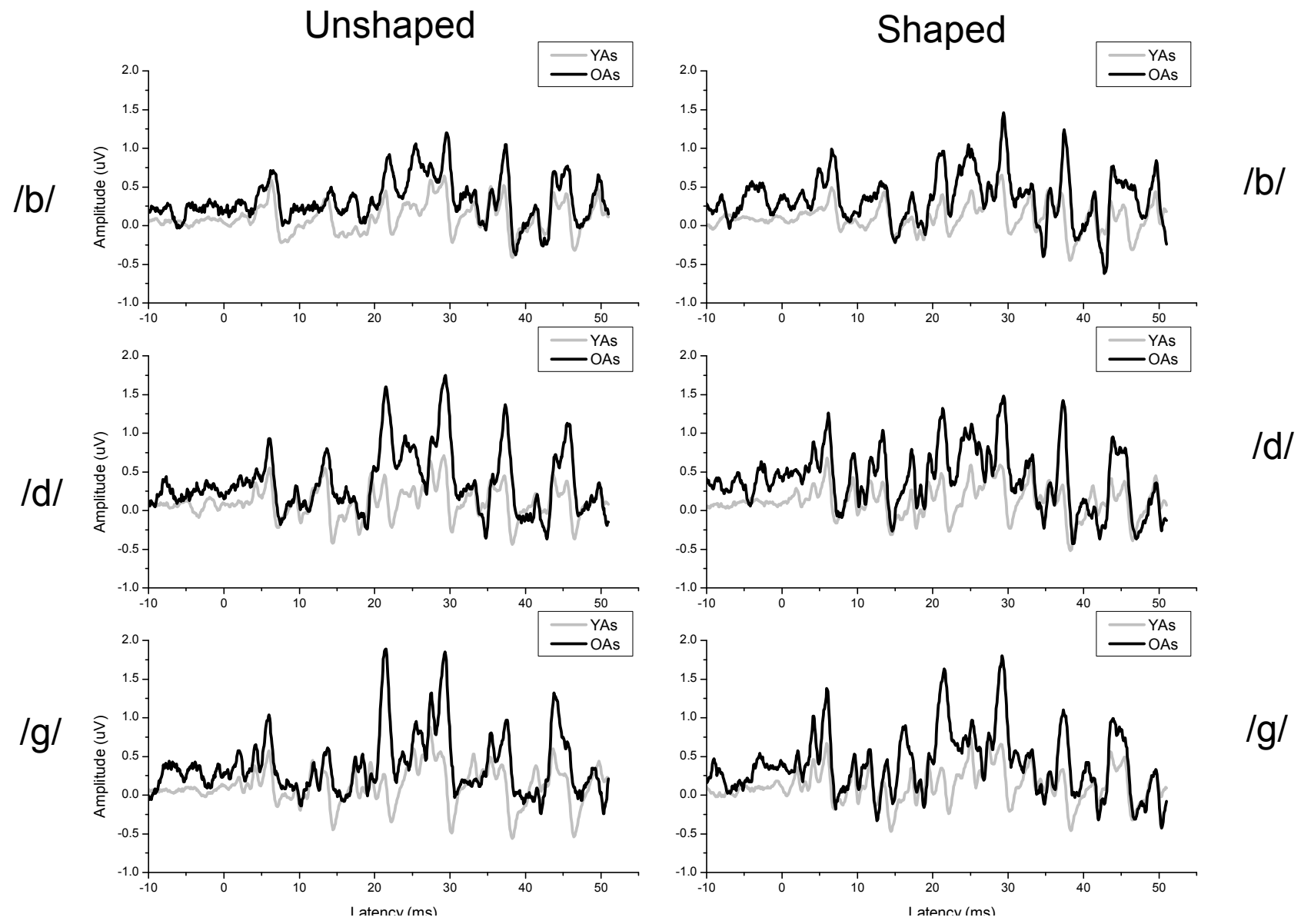

Figure 4-3. Grand Average Waveforms for Young and Older Adults to Unshaped and Shaped Phonemes.

Grand average waveforms for younger and older adults (older adults are plotted in bold) to unshaped (left panels) and shaped (right panels) stimuli/phonemes [/b/,/d/, /g/ are shown in top, middle, and bottom panels, respectively]. 


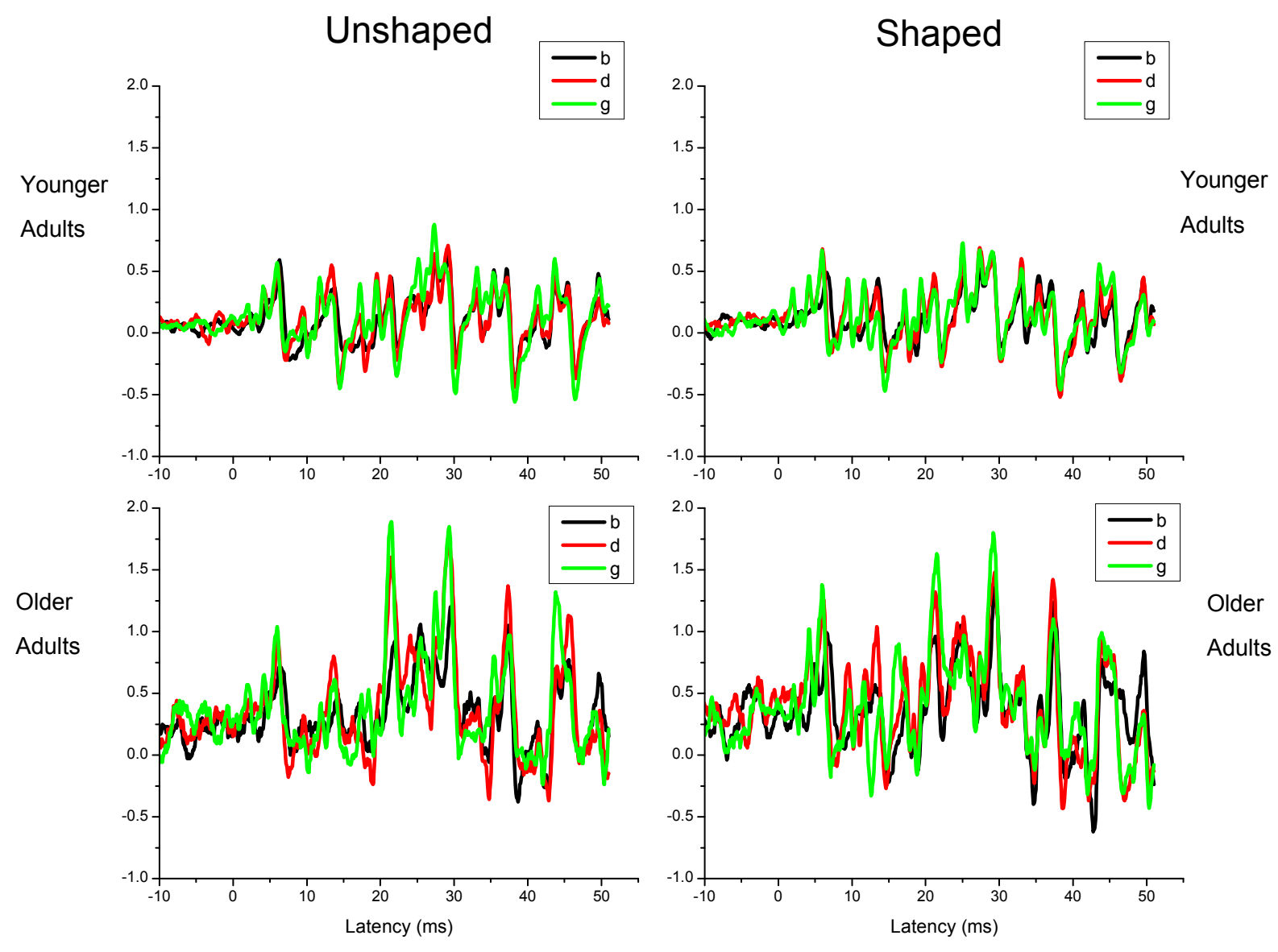

Figure 4-4. Grand Average Waveforms Evoked by the Three Phonemes Are Shown Superimposed for Each Group.

Three superimposed grand average waveforms evoked by the three phonemes (/b/ are plotted in black, /d/ in red, and /g/ in green) for every group (Upper panels for younger adults and lower panels for older adults) and for both shaping conditions (left panels for the shaped stimuli and right panels for the shaped ones). 
Table 4-3. Means and Standard Deviations for All of the Speech-ABR Peaks.

\begin{tabular}{|c|c|c|c|c|c|c|c|c|c|c|c|c|}
\hline \multirow{3}{*}{$\begin{array}{l}\text { Peak } \\
\text { Label }\end{array}$} & \multicolumn{4}{|c|}{$/ \mathbf{b a} /$} & \multicolumn{4}{|c|}{$/ \mathrm{da} /$} & \multicolumn{4}{|c|}{$/ g a /$} \\
\hline & \multicolumn{2}{|c|}{$\begin{array}{c}\text { Latency } \\
\text { (Unshaped) }\end{array}$} & \multicolumn{2}{|c|}{$\begin{array}{c}\text { Latency } \\
\text { (Shaped) }\end{array}$} & \multicolumn{2}{|c|}{$\begin{array}{c}\text { Latency } \\
\text { (Unshaped) }\end{array}$} & \multicolumn{2}{|c|}{$\begin{array}{c}\text { Latency } \\
\text { (Shaped) }\end{array}$} & \multicolumn{2}{|c|}{$\begin{array}{c}\text { Latency } \\
\text { (Unshaped) }\end{array}$} & \multicolumn{2}{|c|}{$\begin{array}{c}\text { Latency } \\
\text { (Shaped) }\end{array}$} \\
\hline & Mean & SD & Mean & SD & Mean & SD & Mean & SD & Mean & SD & Mean & SD \\
\hline 1 & 6.52 & 0.32 & 6.70 & 0.25 & 6.09 & 0.20 & 6.12 & 0.27 & 6.07 & 0.37 & 6.06 & 0.29 \\
\hline 2 & 13.74 & 0.66 & 13.53 & 0.38 & 13.31 & 0.52 & 13.20 & 0.63 & 13.43 & 1.35 & 13.50 & 1.20 \\
\hline 3 & 21.46 & 0.29 & 21.22 & 0.59 & 21.03 & 0.77 & 21.05 & 0.51 & 21.07 & 0.99 & 20.98 & 0.96 \\
\hline 4 & 29.12 & 0.55 & 29.05 & 0.56 & 28.90 & 0.73 & 28.95 & 0.57 & 28.61 & 0.82 & 28.76 & 0.81 \\
\hline 5 & 37.19 & 0.24 & 36.97 & 0.43 & 37.06 & 0.62 & 37.13 & 1.00 & 36.78 & 0.70 & 37.01 & 0.70 \\
\hline 6 & 45.25 & 0.62 & 45.01 & 0.77 & 45.12 & 0.81 & 45.23 & 0.61 & 45.10 & 0.81 & 45.15 & 0.74 \\
\hline (1) & 7.49 & 0.40 & 7.68 & 0.27 & 6.99 & 0.34 & 7.00 & 0.30 & 7.00 & 0.39 & 6.90 & 0.29 \\
\hline (2) & 15.18 & 0.64 & 14.85 & 0.66 & 14.52 & 0.44 & 14.49 & 0.53 & 14.91 & 1.19 & 14.77 & 1.29 \\
\hline (3) & 22.40 & 0.33 & 22.14 & 0.66 & 22.34 & 1.17 & 22.50 & 1.23 & 22.37 & 1.38 & 22.40 & 1.40 \\
\hline (4) & 30.58 & 1.02 & 30.47 & 1.16 & 30.39 & 1.41 & 30.45 & 1.10 & 30.33 & 1.34 & 30.35 & 1.49 \\
\hline (5) & 38.46 & 1.23 & 38.49 & 1.27 & 38.54 & 1.26 & 38.55 & 1.32 & 38.58 & 1.28 & 38.65 & 1.23 \\
\hline (6) & 46.75 & 1.29 & 46.66 & 1.29 & 46.76 & 1.24 & 46.79 & 1.24 & 46.81 & 1.10 & 47.90 & 4.55 \\
\hline b & 18.50 & 0.74 & 18.46 & 0.66 & 18.34 & 0.71 & 18.32 & 0.63 & 18.64 & 1.19 & 18.45 & 1.16 \\
\hline c & 26.27 & 0.91 & 26.11 & 0.97 & 26.26 & 0.77 & 26.17 & 0.85 & 26.28 & 0.77 & 26.20 & 0.84 \\
\hline d & 34.00 & 0.86 & 33.98 & 0.72 & 33.99 & 0.71 & 34.03 & 0.69 & 34.10 & 0.87 & 34.18 & 0.79 \\
\hline 1 & 6.79 & 0.46 & 6.92 & 0.39 & 6.17 & 0.37 & 6.31 & 0.51 & 6.60 & 0.76 & 11.67 & 17.39 \\
\hline 2 & 13.61 & 0.87 & 13.81 & 0.70 & 13.71 & 0.35 & 13.47 & 0.76 & 13.43 & 0.70 & 13.49 & 1.10 \\
\hline 3 & 21.61 & 0.41 & 21.23 & 0.50 & 21.51 & 0.26 & 20.98 & 0.88 & 21.17 & 0.94 & 21.14 & 1.00 \\
\hline 4 & 29.59 & 0.35 & 29.42 & 0.25 & 29.22 & 0.49 & 29.30 & 0.44 & 29.26 & 0.33 & 29.32 & 0.31 \\
\hline 5 & 37.24 & 0.51 & 37.02 & 0.73 & 37.32 & 0.55 & 37.29 & 0.56 & 37.14 & 0.90 & 37.27 & 0.80 \\
\hline 6 & 45.38 & 0.87 & 45.65 & 0.68 & 45.48 & 0.57 & 45.39 & 0.77 & 45.29 & 0.73 & 45.75 & 1.14 \\
\hline (1) & 7.88 & 0.58 & 8.32 & 0.89 & 7.40 & 0.89 & 7.24 & 0.76 & 7.53 & 0.98 & 7.25 & 0.87 \\
\hline (2) & 14.91 & 0.82 & 15.07 & 0.67 & 14.81 & 0.59 & 14.77 & 0.65 & 15.14 & 0.62 & 15.10 & 1.41 \\
\hline (3) & 23.00 & 0.73 & 22.50 & 0.74 & 22.62 & 0.60 & 22.32 & 0.98 & 22.64 & 1.20 & 22.63 & 1.27 \\
\hline
\end{tabular}


Table 4-3. (Continued).

\begin{tabular}{|c|c|c|c|c|c|c|c|c|c|c|c|c|}
\hline \multirow{3}{*}{$\begin{array}{l}\text { Peak } \\
\text { Label }\end{array}$} & \multicolumn{4}{|c|}{$/ \mathbf{b a} /$} & \multicolumn{4}{|c|}{$/ \mathbf{d a} /$} & \multicolumn{4}{|c|}{$/ g a /$} \\
\hline & \multicolumn{2}{|c|}{$\begin{array}{c}\text { Latency } \\
\text { (Unshaped) }\end{array}$} & \multicolumn{2}{|c|}{$\begin{array}{l}\text { Latency } \\
\text { (Shaped) }\end{array}$} & \multicolumn{2}{|c|}{$\begin{array}{c}\text { Latency } \\
\text { (Unshaped) }\end{array}$} & \multicolumn{2}{|c|}{$\begin{array}{l}\text { Latency } \\
\text { (Shaped) }\end{array}$} & \multicolumn{2}{|c|}{$\begin{array}{c}\text { Latency } \\
\text { (Unshaped) }\end{array}$} & \multicolumn{2}{|c|}{$\begin{array}{l}\text { Latency } \\
\text { (Shaped) }\end{array}$} \\
\hline & Mean & SD & Mean & SD & Mean & SD & Mean & SD & Mean & SD & Mean & SD \\
\hline (4) & 30.90 & 0.92 & 30.95 & 0.89 & 30.56 & 0.67 & 30.57 & 0.76 & 30.68 & 0.84 & 30.58 & 0.83 \\
\hline (5) & 38.92 & 1.23 & 39.08 & 1.01 & 38.68 & 0.74 & 38.67 & 0.79 & 38.92 & 1.21 & 38.94 & 0.97 \\
\hline (6) & 46.76 & 0.87 & 47.15 & 0.93 & 46.75 & 0.96 & 48.69 & 5.47 & 47.03 & 1.00 & 47.12 & 1.18 \\
\hline b & 18.53 & 0.46 & 18.33 & 0.55 & 18.27 & 0.69 & 18.44 & 0.89 & 18.35 & 1.17 & 17.84 & 1.15 \\
\hline c & 26.65 & 1.24 & 26.62 & 1.13 & 26.46 & 1.31 & 26.29 & 1.43 & 26.26 & 1.27 & 26.08 & 1.34 \\
\hline d & 34.14 & 0.45 & 34.18 & 0.80 & 34.25 & 0.83 & 34.38 & 0.79 & 34.05 & 1.07 & 34.17 & 0.94 \\
\hline
\end{tabular}

NOTE: Latencies obtained from younger adults are highlighted in grey, whereas those of older adults are not. 


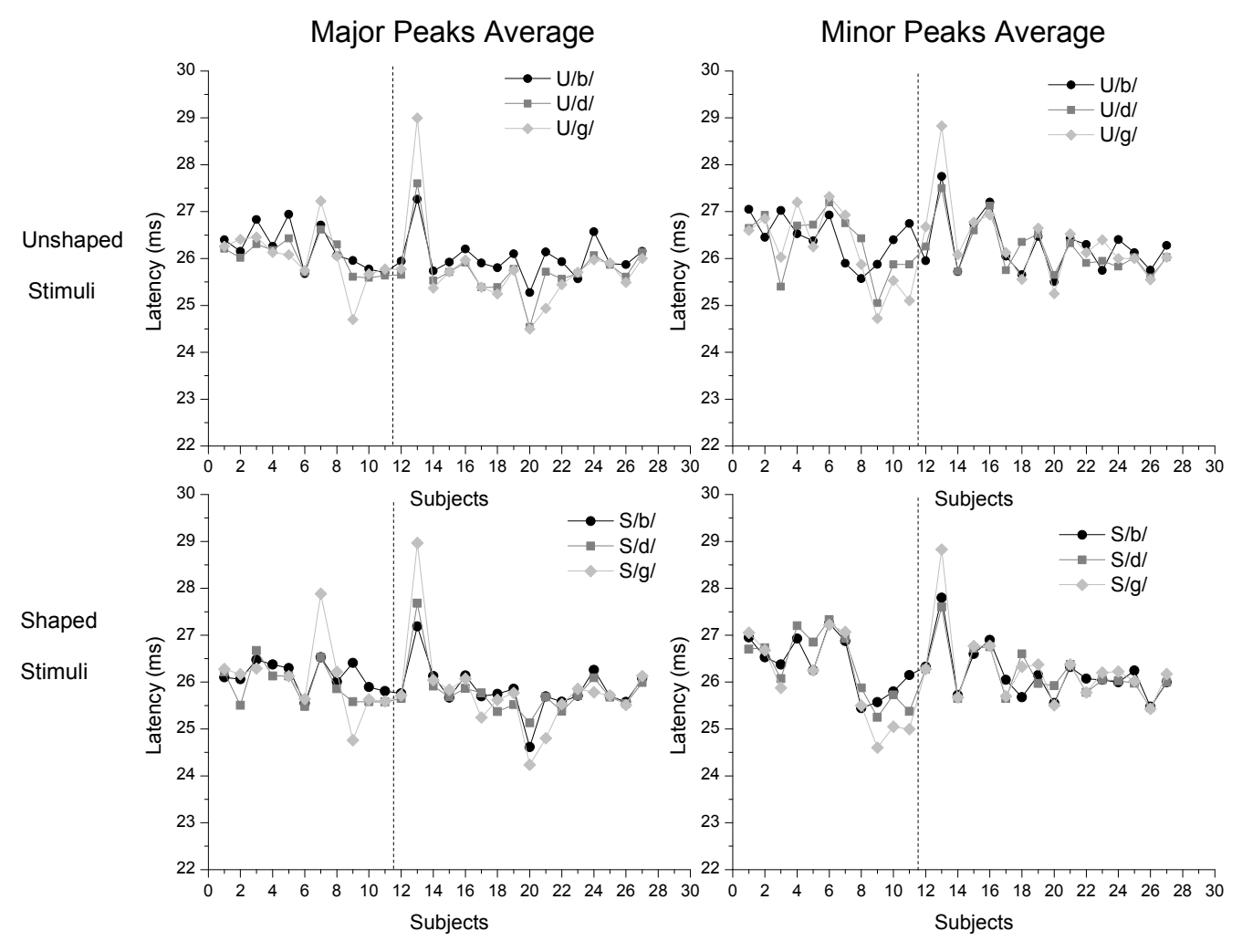

Figure 4-5. Average Latency across All Major and Minor Peaks to Unshaped and Shaped Phonemes for Each Individual Subject.

Average latency across all major (left panels) and minor (right panels) peaks for each phoneme (filled circles for /b/, squares for /d/ and the diamond shape for $/ \mathrm{g} /$ ), for each shaping condition (upper panels for unshaped stimuli, and lower panels for shaped stimuli), for each subject. A dashed vertical line separates younger from older adults. Subjects numbered from 1- 11 are older adults, whereas subjects numbered 12 till 27 are younger adults. 


\section{Stop Consonant Differentiation Score}

The average differentiation scores and standard deviations for each shaping condition and each group are displayed in Table 4-4. The large standard deviations shown in Table 4-4 are indicative of a widely-spread range of differentiation scores for both major and minor peaks. This sub-cortical variability may be attributed to different language experiences of our subjects. Sub-cortical processing is linked to a wide range of reading ability and phonological awareness skills (Hornickel et al. 2009). In other words, the plasticity of the speech-ABR is dependent upon experience (Hornickel, Knowles \& Kraus 2012) and seems to contribute toward this variability of scores.

Two -factor ANOVA was carried out on each differentiation score (i.e. major peaks score and minor peak score) with repeated measures. The factors were shaping condition (unshaped vs. shaped) and age (younger vs. older).

For the differentiation score of major peaks, there were no obtained significant group differences between younger and older adults $[\mathrm{F}(1,24)=0.169, p=0.685]$. However, a significant shaping by group interaction was reported $[\mathrm{F}(1,24)=9.572, p<$ $0.001]$, such that shaping reduced the differentiation score of major peaks in younger adults and increased it in that of older adults.

For the differentiation score of minor peaks, there were no obtained significant group differences between younger and older adults $[\mathrm{F}(1,24)=0.833, p=0.371]$. Not significant were the main effects for shaping condition on the score of minor peaks or any interaction.

In order to evaluate correlations between behavioral and electrophysiological data, two separate differentiation scores were computed for each of the major and minor peaks for each phoneme pair (i.e. /ba/ vs. /da/, /ba/ vs. /ga/, and /da/ vs. /ga/). Likewise, differences in the behavioral area under the curve measure between each phoneme within the same pairs (as above) were computed. Correlations were assessed among these computed pair measurements. Table 4-5 presents a correlation matrix for these pair measurements. In overall, per the correlation assessment, minor peak scores were correlated with major peak scores for shaped pairs, indicating the possible influence of

Table 4-4. Average Differentiation Scores and Standard Deviations.

\begin{tabular}{cccccc}
\hline Group & \multicolumn{2}{c}{ Major Peaks } & & \multicolumn{2}{c}{ Minor Peaks } \\
\cline { 2 - 3 } \cline { 5 - 6 } & Unshaped & Shaped & & Unshaped & Shaped \\
\hline Younger Adults & & & & & \\
Mean & 13.25 & 7.94 & & 5.81 & 3.81 \\
SD & 11.41 & 10.18 & & 5.62 & 3.76 \\
Older Adults & & & & 6.00 & 6.60 \\
Mean & 6.20 & 11.70 & & 6.38 & 4.79 \\
SD & 9.00 & 12.46 & &
\end{tabular}


Table 4-5. Correlation Matrix for Behavioral and Electrophysiological Phoneme Pairs.

\begin{tabular}{|c|c|c|c|c|c|c|c|c|c|c|c|c|c|c|c|c|c|}
\hline \multirow[b]{2}{*}{ Stimuli } & \multicolumn{5}{|c|}{ Major Peaks Score for } & \multicolumn{6}{|c|}{ Minor Peaks Score for Pairs } & \multicolumn{6}{|c|}{ Differences in Area Under the Curve Measure for Pairs } \\
\hline & Ub-g ${ }^{1}$ & Ud-g ${ }^{1}$ & $S b-d^{1}$ & Sb-g ${ }^{1}$ & Sd-g ${ }^{1}$ & $\overline{\text { Ub-d }}{ }^{2}$ & Ub-g ${ }^{2}$ & Ud-g ${ }^{2}$ & Sb-d ${ }^{2}$ & $\mathbf{S b}-\mathbf{g}^{2}$ & $\overline{S d-g^{2}}$ & $\mathrm{Ub}^{-d^{3}}$ & $\mathrm{Ub}-\mathrm{g}^{3}$ & Ud-g ${ }^{3}$ & Sb-d ${ }^{3}$ & Sb-g ${ }^{3}$ & Sd-g ${ }^{3}$ \\
\hline Ub-d ${ }^{1}$ & $0.627 * *$ & 0.103 & 0.013 & 0.366 & $0.437^{*}$ & $0.392 *$ & 0.202 & -0.222 & 0.238 & 0.109 & 0.092 & -0.104 & -0.093 & -0.012 & -0.058 & -0.181 & -0.128 \\
\hline Ub-g ${ }^{1}$ & & $0.731^{* *}$ & 0.117 & $0.65^{* *}$ & $0.588^{* *}$ & 0.284 & 0.281 & 0.106 & -0.028 & 0.094 & 0.299 & -0.100 & -0.318 & -0.203 & 0.001 & -0.274 & -0.236 \\
\hline Ud-g ${ }^{1}$ & & & 0.175 & $0.591^{* *}$ & $0.430^{*}$ & 0.062 & 0.095 & 0.205 & -0.047 & 0.097 & 0.53 & 0.004 & -0.152 & -0.130 & -0.011 & -0.054 & -0.016 \\
\hline$S b-d^{1}$ & & & & $0.490^{*}$ & -0.109 & -0.268 & -0.083 & $0.448^{*}$ & 0.339 & $0.412^{*}$ & 0.270 & -0.078 & 0.109 & 0.168 & 0.108 & 0.234 & 0.112 \\
\hline Sb-g ${ }^{1}$ & & & & & $0.688^{* *}$ & 0.120 & 0.036 & 0.160 & $0.420^{*}$ & $0.579^{* *}$ & 0.308 & -0.166 & -0.249 & -0.089 & 0.096 & -0.118 & -0.014 \\
\hline Sd-g ${ }^{1}$ & & & & & & $0.458^{*}$ & 0.231 & -0.196 & 0.236 & $0.576^{* *}$ & 0.234 & -0.299 & -0.333 & -0.060 & $-0.408^{*}$ & -0.285 & 0.123 \\
\hline Ub-d ${ }^{2}$ & & & & & & & $0.720^{* *}$ & -0.068 & 0.225 & $0.394 *$ & 0.027 & 0.032 & -0.102 & -0.093 & -0.163 & -0.339 & -0.097 \\
\hline Ub-g ${ }^{2}$ & & & & & & & & $0.393 *$ & -0.032 & 0.294 & 0.007 & 0.123 & -0.238 & -0.292 & -0.004 & $-0.410^{*}$ & -0.302 \\
\hline Ud-g ${ }^{2}$ & & & & & & & & & -0.096 & 0.125 & $0.415^{*}$ & 0.154 & -0.106 & -0.211 & 0.099 & -0.079 & -0.138 \\
\hline Sb-d ${ }^{2}$ & & & & & & & & & & $0.585^{* *}$ & -0.003 & -0.133 & 0.251 & 0.311 & 0.058 & 0.211 & 0.113 \\
\hline Sb-g ${ }^{2}$ & & & & & & & & & & & 0.351 & -0.140 & 0.002 & 0.119 & -0.165 & 0.103 & 0.250 \\
\hline Sd- $\mathrm{g}^{2}$ & & & & & & & & & & & & 0.157 & 0.095 & -0.063 & -0.075 & 0.165 & 0.196 \\
\hline Ub-d $\mathbf{d}^{3}$ & & & & & & & & & & & & & 0.359 & $-0.498^{* *}$ & $0.565^{* *}$ & 0.302 & -0.254 \\
\hline Ub-g ${ }^{3}$ & & & & & & & & & & & & & & $0.631^{* *}$ & 0.363 & $0.815^{* *}$ & $0.393 *$ \\
\hline Ud-g ${ }^{3}$ & & & & & & & & & & & & & & & -0.126 & $0.506^{* *}$ & $0.576^{* *}$ \\
\hline$S b-d^{3}$ & & & & & & & & & & & & & & & & $0.414^{*}$ & $-0.569^{* *}$ \\
\hline Sb-g ${ }^{3}$ & & & & & & & & & & & & & & & & & $0.512^{* *}$ \\
\hline Sd-g ${ }^{3}$ & & & & & & & & & & & & & & & & & \\
\hline
\end{tabular}

NOTES: This table shows correlations among sub-cortical differentiation scores for major ${ }^{1}$ and minor ${ }^{2}$ peaks of six /b-d-g/ phoneme pairs as well as among differences ${ }^{3}$ in the behavioral area under the curve measure between phonemes of each pair. The " $U$ " and "S" letters plotted before all pairs indicate the unshaped and shaped conditions, respectively.

Measures in this table were considered strongly correlated if $\mathrm{r}= \pm 0.30$ and $* P \leq 0.05 ; * * P \leq 0.01$. 
shaping on both types of waves; major and minor peaks. Major peak scores of unshaped pairs were correlated with each other but not with that of minor peaks, suggesting that minor and major peaks may underlie different biological contributors. Furthermore, behavioral differences in area under the curve measure were correlated with each other but not with any of the electrophysiological scores showing no relations between the behavioral results and the sub-cortical responses.

\section{Absolute Amplitudes}

A 3 X 2 X K X 2 repeated measures ANOVA was performed on the absolute amplitudes of onset peaks, major peaks, minor peaks, and endpoint peaks, separately, where $\mathrm{K}$ denotes the number of peaks. The factors were phoneme (repeated measures on 3 levels, /b/ vs. /d/ vs. /g/), shaping condition (repeated measures on 2 levels, shaped vs. unshaped), peaks (repeated measures on K levels: 2 for onset peaks, 8 for major peaks, 3 for minor peaks and 2 for endpoint peaks), and age ( 2 levels, young vs. old).

For the amplitudes of onset peaks, there were no obtained group differences between younger and older adults $[\mathrm{F}(1,24)=3.379, p=0.078]$. A significant main effect for phoneme $[\mathrm{F}(2,23)=3.600, p=0.035]$ and for peak $\mathrm{F}(1,24)=19.060, p<0.001]$ were reported, such that the amplitudes of onset peaks for the $/ \mathrm{d} /$ phoneme were larger than that for the $/ \mathrm{b} /$ and $/ \mathrm{g} /$ phonemes. Also, the amplitude of the positive onset peak [i.e. 1] is larger than that of the negative onset peak [i.e. (1)]. A significant phoneme by shaping interaction was found $[\mathrm{F}(2,23)=3.959, p=0.026]$.

To assess the effects of shaping on both onset peaks for each particular phoneme, a follow up paired t-tests was conducted comparing amplitudes of both endpoint peaks (collapsed all over the 2 peaks) before and after the introduction of shaping, for each one of the three phonemes. In overall, shaping increased the amplitudes of onset peaks for the $/ \mathrm{g} /$ phoneme $[\mathrm{t}(51)=-2.250, p=0.029]$, in particular.

As for the amplitudes of endpoint peaks, there were no obtained significant group differences between younger and older adults $[\mathrm{F}(1,24)=0.201, p=0.658]$. Also, there were no obtained significant main effects for any of the factors or any significant interaction.

As for the amplitudes of major peaks, there were no obtained significant group differences between younger and older adults $[\mathrm{F}(1,24)=1.525, p=0.0 .229]$. However, multiple significant main effects and interactions were found. A significant main effect for phoneme was reported $[\mathrm{F}(2,23)=7.599, p=0.001]$, indicating that amplitudes of major peaks increased from $/ \mathrm{b} /$ to $/ \mathrm{d} /$ to $/ \mathrm{g} /$. A significant main effect for peak was reported $[\mathrm{F}(2.381,57.138)=5.340, p=0.005]$, such that amplitudes increased from wave 2 to 3 to 4, respectively, and dropped for 5, (2) and (3) and started to increase back for peaks (4) and (5). A significant phoneme by peak interaction was found [F (5.208, $125.004)=2.932, p=0.014]$. Follow up paired t-tests were conducted to evaluate differences among phonemes for each peak, and results (only that were found to be 
significant) are shown in Table 4-6. In this table, amplitudes increased from $/ \mathrm{b} /$ to $/ \mathrm{d} /$ phonemes and from /b/ to /g/ phonemes in the (ba vs. da) and (ba vs. ga) pairs, respectively.

A significant phoneme by peak by group interaction was also found $[\mathrm{F}(14,280)=$ 2.829, $p=0.001]$. Separately for each group, a follow-up 2-way ANOVA with phoneme and peak as factors was performed on the major peaks' amplitudes (collapsed all over the two shaping conditions). For the younger adult group, a significant 2-way (phoneme $\mathrm{X}$ peak) interaction $[\mathrm{F}(7.028,217.879)=5.304, p<0.001]$ was obtained. There was no significant 2-way (phoneme X peak) interaction $[\mathrm{F}(4.848,96.963)=1.699, p=0.144]$ obtained for older adults. Consequently, paired t-tests were conducted to evaluate differences among phonemes for each peak in younger adults, results (only that were found to be significant) are shown in Table 4-7. Amplitudes increased from $/ \mathrm{b} /$ to $/ \mathrm{d} /$ phonemes and from /b/ to /g/ phonemes in the (ba vs. da) and (ba vs. ga) pairs, respectively.

A significant phoneme by shaping by peak was reported $[\mathrm{F}(5.784,138.810)=$ $2.454, p=0.029]$. For each peak, a follow-up 2-way ANOVA with shaping condition and phoneme as factors was performed on the peaks' amplitudes. There were no significant 2way interactions obtained for any one of the major peaks.

As for the amplitudes of minor peaks, there were no obtained significant group differences between younger and older adults $[\mathrm{F}(1,24)=0.144, p=0.708]$. However, a significant main effect for phoneme $[\mathrm{F}(2,23)=4.525, p=0.016]$ and for peak $[\mathrm{F}(1.547$, $37.122)=7.918, p=0.003]$ were reported, such that amplitudes of minor peaks increased from $/ \mathrm{b} /$ to $/ \mathrm{d} /$ to $/ \mathrm{g} /$ phonemes. It also indicated that amplitudes of waves " $\mathrm{b}$ " and " $\mathrm{d}$ " were larger than that of wave "c". Additionally, a significant peak by group interaction was reported $[\mathrm{F}(2,23)=2.454, p=0.029]$. To assess group differences for each peak, amplitudes were collapsed for all phonemes and shaping conditions and were compared between younger and older adults using a paired t-tests. The amplitude of minor peak $d$ was significantly [ $\mathrm{t}(64)=2.901, p=0.005]$ larger for older adults than for younger adults.

As for the amplitudes of minor peaks, there were no obtained significant group differences between younger and older adults $[\mathrm{F}(1,24)=0.144, p=0.708]$. However, a significant main effect for phoneme $[\mathrm{F}(2,23)=4.525, p=0.016]$ and for peak $[\mathrm{F}(1.547$, $37.122)=7.918, p=0.003]$ were reported, such that amplitudes of minor peaks increased from $/ \mathrm{b} /$ to $/ \mathrm{d} /$ to $/ \mathrm{g} /$ phonemes. It also indicated that amplitudes of waves " $\mathrm{b}$ " and " $\mathrm{d}$ " were larger than that of wave "c". Additionally, a significant peak by group interaction was reported $[\mathrm{F}(2,23)=2.454, p=0.029]$. To assess group differences for each peak, amplitudes were collapsed for all phonemes and shaping conditions and were compared between younger and older adults using a paired t-tests. The amplitude of minor peak $\mathrm{d}$ was significantly $[\mathrm{t}(64)=2.901, p=0.005]$ larger for older adults than for younger adults. 
Table 4-6. Significant Results of Paired T-tests for Amplitude Differences among Phoneme Pairs.

\begin{tabular}{|c|c|c|c|c|c|c|c|c|c|c|}
\hline \multirow[t]{2}{*}{ Pair } & \multicolumn{2}{|c|}{ Wave 3} & \multicolumn{2}{|c|}{ Wave 4} & \multicolumn{2}{|c|}{ Wave (2) } & \multicolumn{2}{|c|}{ Wave (3) } & \multicolumn{2}{|c|}{ Wave (4) } \\
\hline & $\mathbf{t}$ & $\mathbf{p}$ & $\mathbf{t}$ & $\mathbf{p}$ & $\mathbf{t}$ & p & $\mathbf{t}$ & $\mathbf{p}$ & $\mathbf{t}$ & $\mathbf{p}$ \\
\hline ba vs. da & -2.032 & 0.047 & N/A & N/A & -4.240 & $<0.001$ & -4.288 & $<0.001$ & -4.819 & $<0.001$ \\
\hline ba vs. ga & -3.040 & 0.004 & -2.554 & 0.014 & -5.560 & $<0.001$ & -4.137 & $<0.001$ & -5.305 & $<0.001$ \\
\hline
\end{tabular}

Table 4-7. Significant Results of Paired T-tests for Amplitude Differences among Phoneme Pairs in Younger Adults.

\begin{tabular}{|c|c|c|c|c|c|c|c|c|c|c|c|c|}
\hline \multirow[t]{2}{*}{ Pair } & \multicolumn{2}{|c|}{ Wave 3} & \multicolumn{2}{|c|}{ Wave 4} & \multicolumn{2}{|c|}{ Wave (2) } & \multicolumn{2}{|c|}{ Wave (3) } & \multicolumn{2}{|c|}{ Wave (4) } & \multicolumn{2}{|c|}{ Wave (5) } \\
\hline & $\mathbf{t}$ & $\mathbf{p}$ & $\mathbf{t}$ & $\mathbf{p}$ & $\mathbf{t}$ & $\mathbf{p}$ & $\mathbf{t}$ & $\mathbf{p}$ & $\mathbf{t}$ & $\mathbf{p}$ & $\mathbf{t}$ & $\mathbf{p}$ \\
\hline ba vs. & -2.198 & 0.036 & N/A & $\mathrm{N} / \mathrm{A}$ & -5.121 & $<0.001$ & -4.307 & $<0.001$ & -4.605 & $<0.001$ & N/A & $\mathrm{N} / \mathrm{A}$ \\
\hline $\begin{array}{l}\text { ba vs. } \\
\text { ga }\end{array}$ & N/A & N/A & -2.128 & 0.041 & -6.023 & $<0.001$ & -4.675 & $<0.001$ & -4.935 & $<0.001$ & -2.697 & 0.011 \\
\hline
\end{tabular}




\section{CHAPTER 5. DISCUSSION}

\section{Effects of Age-related Hearing Loss}

The first major objective of this present study was to investigate the effects of aging on the neural representation of contrastive voiced stop consonant stimuli at the brainstem level. The second main objective was to determine whether the introduction of spectral-shaping onto contrastive voiced stop consonant stimuli (as in Harkrider et al. 2005,2006 ) by enhancing the F2 transition reduces potential impairments, if existed, in the sub-cortical neural representations and the behavioral identification of these stimuli in older adults. To do so, responses were obtained from younger and older adults with normal to near normal hearing at the frequencies most dominant in the F2 formant transitions of the $\mathrm{CV}$ stimuli.

The essential feature for the perception of phonemes used in this current study, i.e. the $\mathrm{F} 2$ transition, ranged from $900 \mathrm{~Hz}$ to $2300 \mathrm{~Hz}$. Older adult subjects had normal to near normal hearing levels at this frequency range and group differences in threshold were not significant until $2000 \mathrm{~Hz}$. Moreover, the stimuli were presented at suprathreshold levels and were adequately audible to all older adult subjects.

Furthermore, it is worth noting that the investigators of this current study repeated all of the exact analyses employed in assessing the collected data without including the responses of the four older adults with increased thresholds. These analyses yielded similar results to what reported here in this current study. Due to the huge amount of data analyses and its similarity to what is reported in the result section, the results of these repeated analyses were not described in this paper.

\section{Effects of Aging}

In this current study, older adults had larger areas under the curve than younger adults for all of the three phonemes (Figure 4-1). Results suggest that our older adult group had more robust categorical perception, or better defined categories, than the younger adults recruited in this present study. Nevertheless, phoneme categories were generally well-defined in our younger adults also. The responses of the $/ \mathrm{b} /$ phoneme were around $10 \%$ or less for tokens at the $/ \mathrm{g} /$ region of the continuum, whereas, the responses of the $/ \mathrm{g} /$ phoneme were less than $10 \%$ for tokens at the $/ \mathrm{b} /$ region of the continuum. As for the $/ \mathrm{d} /$ category, responses of the /d/ phoneme were less than $20 \%$ for tokens at both end regions of the continuum (Dorman et al. 1985).

Several studies reported impaired performance on stop consonant identification tasks in normal-hearing older adults (e.g. Dorman et al. 1985; Harkrider et al. 2005; Harkrider et al. 2006). Despite using the same behavioral methodology employed in the aforementioned studies, our older adults did not show similar stop consonant perception deficits. However, the normal performance on stop consonant identification of older 
adults in this present study is consistent with some previous findings reported in literature. For example, in a study by Helfer and Huntley (1991), consonant identification accuracy and error patterns were examined in older adults with minimal hearing loss using the City University of New York (CUNY) nonsense syllables. Their minimally- hearing impaired older adults performed normally "in quiet" when compared to "in noise" conditions. In spite of many documented stop consonant impairments in normal-hearing older adults, many of this population seem to perform normally in stop consonant identification tasks in quiet listening environments. Aging does not seem to affect consonant error patterns or cause unique confusion patterns (Helfer and Huntley 1991), thus, performance on consonant identification tasks might vary among older adults. In another study by Wong, Jin, Gunasekera, Abel, Lee and Dhar (2008), normalhearing older adults showed no deficits in speech perception tasks conducted in quiet as well. In this study, however, the normal-hearing older adults identified single word stimuli taken from Raymer, Maher, Greenwald, Morris, Rothi, and Heilman (1990). Similar to the aforementioned two latter studies (i.e. Hefler \& Huntley 1991 and Wong et al. 2008), many other studies, which evaluated the influence of age on speech perception in noise, assessed the performance of their normal-hearing or minimally hearing-impaired older adults on speech perception tasks in quiet with no background noise as a point of reference. Several of these studies reported normal speech perception in their quiet experimental conditions (e.g. Tun 1998; Stuart \& Phillips 1996; Gordon-Salant 1987). It is worth noting that Tun (1998) used time compressed spoken sentences, whereas Stuart and Phillips (1996) and Gordon-Salant (1987) used monosyllabic word materials. In another relevant study by Surprenant (2007), normal-hearing older adults even performed normally or as equally as normal-hearing young listeners in a speech identifying task that used six consonant-vowel syllables (i.e. $/ \mathrm{ba} /, / \mathrm{da} /, / \mathrm{ga} /, / \mathrm{ka} /, / \mathrm{pa} /$ and $/ \mathrm{ta} /$ ) $\mathrm{presented}$ with broadband noise at a $0,+5$ and +25 signal to noise ratios (SNR). There were no age differences reported between normal-hearing older and younger adults in that study, however, once noise was increased, age differences started to emerge.

Despite the normal processing of single words in quiet, normal-hearing older adults of Wong et al. (2008) study performed more poorly than younger adults in identifying single words in multi-talker babble noise with a SNR of $-5 \mathrm{~dB}$. For this noisy condition, older adults of this study showed, on fMRI examinations, a decline in auditory cortex activation, but also an increase in cortical activities in general cognitive areas that positively correlated with their speech perception performance. Such observations of brain images support the decline-compensation hypothesis in older adults for the auditory domain (Wong et al. 2008). In other words, the brains of older adults appear to increase activation in general cognitive (association) areas to counteract the age-related reductions in the activation of sensory regions. In normal-hearing aged listeners, the listening condition of the behavioral measures employed in this present study and many other speech perception tasks performed in quiet might be less demanding at the cognitive level than complex listening tasks such as identifying speech in noisy or reverberant environments (Martin \& Jerger 2005). Presenting speech in noise or in any other degrading listening situations (e.g. compressed speech) will tax the cognitive processing resources of older adults, despite their initially perfect speech identification (Surprenant 2007). During taxed listening environments, older adults might need to summon all 
possible cognitive resources assigned to process speech in a manner that will dictate their perceptual efficiency (Surprenant 2007). Nevertheless, this will be restricted to tasks that are mediated by top-down processes where the given task critically depends on the true and appropriate identification of the tested stimulus itself (Surprenant 2007). Some older adults, in general, may show substantial cognitive impairments and some other may perform as well as younger adults or even better (Cabeza, Anderson, Locantore, and McIntosh 2002). This variability in cognitive abilities might attribute to the differences in speech perception abilities among older adults.

In this current study, the aggregate speech- ABR was used to investigate age effects on the differentiation of stop-consonant phonemes at the sub-cortical level in older listeners with normal to near normal hearing. Previous studies originally reported impaired cortical evoked potentials elicited by similar phonemic contrasts in older listeners regardless of hearing status. Moreover, normal-hearing older adults showed timing and amplitude abnormalities at the offset and onset parts of the speech-ABR evoked by a 40-ms /da/, respectively (Vander Werff \& Burns 2011).The exact same stop consonant stimuli (both unshaped and shaped) of this current investigation were originally used in Harkrider et al. (2005) study to evoke obligatory N1-P2 cortical responses in normal-hearing older adult population. Prolonged P2 latencies and larger N1 amplitudes were obtained for the unshaped stimuli in their older adults. However, P2 latencies were more similar to those of younger adults once spectral-shaping was introduced onto the stimuli. N1 amplitudes remained larger in older adults for the spectrally-shaped stimuli. Again, the same unshaped and shaped stimuli were used in Harkrider et al. (2006), but this time to record the N1-P2 cortical responses in older adults with mild to moderate hearing loss in addition to normal hearing older adults. When compared to normal hearing younger adults, their older adults showed larger N1 and P2 amplitudes and prolonged P2 latencies regardless of their hearing level.

Two electrophysiological differences were obtained between our older and younger adults using the experiment protocols of this current study. One difference is the amplitude of peak " $d$ ", which is one of the minor peaks of the speech-ABR evoked by a 170-ms CV syllable. Older adults showed greater amplitudes for their minor wave "d" than younger adults. The other difference is the amplitude of the negative major peak "(5)". The amplitude of this peak increased from the phoneme /b/ to /d/ and from /b/ to $\mathrm{g} / \mathrm{in}$ younger adults. However, such amplitude-related distinction was not found in older adults. On the other hand, no age-related latency differences were found between the two groups.

Although a larger wave " $d$ " amplitude might indicate a negative byproduct of aging, it certainly did not affect the behavioral identification of stop consonants of our older adults. It is possible that the aging auditory brainstem is trying to compensate for impairments in temporal processing by attending to the CV stimuli; a brain activity which can be translated into a generalized boost in neuronal firing and consequently into larger amplitudes (Harris, Wilson, Eckert, \& Dubno 2012). Declines in inhibitory mediation within the central auditory nervous system, possibly caused by aging, might be another explanation for larger amplitudes in older adults (Harris, Mills \& Dubno 2007). In 
contrast, the distinctly-enhanced amplitudes of the speech-ABR peaks across phonemes in younger adults might reflect an intact sub-cortical differentiating mechanism among the three phonemes. The aging brainstem might have lost this intricate capacity, where a generalized accentuation, received from upper cortical levels, rather than a distinct enhancement may take place at the sub-cortical pathway in the elderly.

The between-stimulus latency distinctions, illustrated in Figure 4-5, demonstrate neural frequency and timing differences differentiating among the $/ \mathrm{b} /, / \mathrm{d} /$, and $/ \mathrm{g} /$ phonemes at the sub-cortical level. Due to the tonotopic distribution of the auditory pathway, latencies of speech-ABR components were expected to be the shortest for the phoneme with the highest F2 onset frequency (i.e. /g/), intermediate for the phoneme with the middle F2 onset frequency (i.e. /d/), and the most prolonged for the phoneme with the lowest F2 onset frequency (i.e./b/). Our individual data (Figure 4-5) showed this expected latency pattern/progression in major peaks for 11 out of 16 younger adults and only 2 out of 11 older adults. In other words, for major peaks, $68 \%$ of our younger adults showed this latency pattern, meanwhile only $18 \%$ of our older adults exhibited this pattern. Such observations suggest age-related abnormalities in the sub-cortical differentiation of stop consonants in our older adult population at the individual level. On the other hand, minor peaks showed this hypothesized latency progression in 4 out of 16 (i.e. $25 \%$ of) younger adults and in 4 out of 11 (i.e. $36 \%$ of) older adults. This additional observation at the individual level supports the notion that the neural generators of minor and major peaks may convey different acoustic information (e.g. Hornickel et al. 2009). As described above, more of our subjects showed the expected latency pattern for major peaks when compared to minor peaks. However, in contrary to our study observation, this systematic latency pattern was detected more in minor rather than in major peaks at the individual level in the studies by Johnson et al. (2008) and Hornickel et al. (2009). According to our individual data, major peaks seem to contribute toward the encoding of filter/formant information that help differentiate among the $/ \mathrm{b}-\mathrm{d}-\mathrm{g} / \mathrm{phonemes}$ to a greater degree than minor peaks. An explanation for such finding differences remains unclear. However, differences in the stimuli used in this present study and the latter two studies might contribute to this inconsistent finding. Table 5-1 shows the major differences between our stimuli and the stimuli used in the latter studies.

The synthetic CV stimuli used in this current study lacked a release of burst whereas that of Johnson et al. (2008) and Hornickel et al. (2009) studies contained an initial burst. It is possible that the presence of the burst improved the identification accuracy of the /b-d-g/ phonemes by enhancing the perception of place of articulation (Blumstein \& Stevens 1980). Such improvement may have been reflected in the speechABR peaks, as a consequence. The trading relation between the burst and the formant transition can provide listeners with the cues for place of articulation perception in stop consonants (Blumstein \& Stevens 1980). Initial investigations that studied the cues contributing to the perception of place of articulation suggested independent roles for onset spectra cues and for the context dependent cue, i.e. the formant transition, in the perception of place of articulation (Blumstein \& Stevens 1980). Earlier investigators even speculated that in spite of the formant transition's primary role in identifying place of articulation and despite its well-studied long history as a major cue in the perception of 
Table 5-1. Stimuli Differences.

\begin{tabular}{lcc}
\hline Stimuli Characteristics & Current Study & Hornickel et al. (2009) \\
\hline $\begin{array}{l}\text { Duration of stimuli } \\
\text { Duration of formant } \\
\text { transition }\end{array}$ & $100 \mathrm{~ms}$ & $170 \mathrm{~ms}$ \\
F1 & $40 \mathrm{~ms}$ & $50 \mathrm{~ms}$ \\
F2 & $150-750 \mathrm{~Hz}$ & $400-720 \mathrm{~Hz}$ \\
& $/ \mathrm{ga} / 2480-1220 \mathrm{~Hz}$ & $/ \mathrm{ga} / 2480-1240 \mathrm{~Hz}$ \\
& $/ \mathrm{da} / 1700-1220 \mathrm{~Hz}$ & $/ \mathrm{da} / 1700-1240 \mathrm{~Hz}$ \\
F3 & $\mathrm{ba} / 900-1220 \mathrm{~Hz}$ & $/ \mathrm{ba} / 900-1240 \mathrm{~Hz}$ \\
F4 & $2400 \mathrm{~Hz}$ & $2850 \mathrm{~Hz}-2500 \mathrm{~Hz}$ \\
Initial Burst & $3300 \mathrm{~Hz}$ & $3300 \mathrm{~Hz}$ \\
\hline
\end{tabular}

place of articulation, onset spectra, alone, can adequately provide information regarding the perception of place of articulation (Blumstein \& Stevens 1980).

Onset spectrum and the final value of the formant transition are two types of acoustic properties that are processed in parallel in order to identify a voiced stop consonant in context, such that the first 10-20 ms of the syllable are considered imperative for the perception of place of articulation (Ohde \& Stevens 1983). When a consonantal release or onset with high frequency properties is followed by a relatively high F2 onset frequency, the spectrum amplitude at frequencies above F2 tends to increase emphasizing the high frequency onset properties (Ohde \& Stevens 1983). Effects of bursts on the differentiation of stop consonant phonemes can be seen even at the physiological level. Delgutte (1980) obtained responses elicited by speech-like noise bursts from nerve fibers at the auditory nerve level. Auditory nerve fibers tend to overshoot their firing targets in response to abrupt onsets which characterize alveolar stops, but tend to undershoot their targets in response to gradual onsets as in the case of labial stops (Ohde \& Stevens 1983).

Furthermore, differences in overall durations may have contributed toward the observed discrepancies found between the findings of this current study and that of Johnson et al. (2008) and Hornickel et al. (2009). In the study by Blumstein and Stevens (1980), identification responses were found to be slightly better for $/ \mathrm{b} /$ and $/ \mathrm{d} / \mathrm{stimuli}$ as opposed to the $/ \mathrm{g} /$ phoneme for shorter overall durations than for longer ones. Longer overall duration may have contributed toward an improvement in the perception of place of articulation and consequently toward an enhanced phoneme differentiation in Johnson et al. (2008) and Hornickel et al. (2009). However, such contribution seems to be minimal due to the small improvement effects that can be attained by longer durations (Blumstein \& Stevens 1980).

Due to the possible influence of the burst on the perception of place of articulation, it is possible that the presence of the burst in Johnson et al. (2008) and Hornickel et al. (2009) enhanced the differentiation of their studied phonemes at the sub- 
cortical level. This enhancement may have been reflected in both major and minor peaks of their subjects' speech-ABRs, mainly in minor peaks. It is possible that minor peaks originate from the distinct interaction between the onset burst and the F2 onset frequency of these phonemes that sub-cortically differentiated among them. Despite aiming to minimize differences among stimuli in order to particularly trace F2 formants into brainwaves, as a sole contributor to the perception of place articulation, the interaction between onset characteristics and F2 value at onset is difficult to rule out. Such interaction may have been encoded in the minor fluctuations of the speech-ABR. Data collected from this current study seem to support this notion, because with the use of unreleased stimuli, the between-stimulus latency distinctions in minor peaks were minimized. In other words, latency distinctions were seen less in minor peaks when unreleased stimuli were utilized, whereas minor peaks showed this pattern of activity with released stimuli in a steady manner as observed in Johnson et al. (2008) and Hornickel et al. (2009) studies. Such finding suggests possible contribution of the burst toward minor peaks.

\section{Effects of Spectral Shaping}

For both groups, shaping increased the behavioral area under the curve for $/ \mathrm{g} /$ phoneme, but decreased it for $/ \mathrm{b} /$ and $/ \mathrm{d} /$ phonemes. Spectral shaping enhances highfrequency information. Therefore, the stimulus with the highest second formant frequency, i.e. $/ \mathrm{g} /$, received the largest amplification, whereas stimuli with low- and midfrequency second formants, i.e. /b/ and /d/, received less amplification. Increased intensities of the stimuli improved subjects' sensitivity to tokens at the $/ \mathrm{g} / \mathrm{phoneme}$ region and resulted in a better defined /g/ category.

Interestingly, spectral shaping appears to enhance the neural differentiation of stop consonant phonemes at the sub-cortical level by improving (i.e. raising) the stop consonant differentiation score of major peaks in our older adults. Differentiating among the phonemic contrasts of stop consonant stimuli is thought to be encoded via systematic latency shifts at the sub-cortical level. In Johnson et al (2008) and Hornickel et al (2009) studies, investigators hypothesized that the $/ \mathrm{b}-\mathrm{d}-\mathrm{g} /$ stop consonants consisting of different frequency components will elicit progressively earlier speech ABR responses for the /g/, $/ \mathrm{d} /$, and $/ \mathrm{b} /$ phonemes, respectively. Investigators speculated that frequency differences among phonemes arising from differences in their places of articulation are encoded in latency shifts based on the tonotopic distribution of the auditory pathway. Findings from both of the latter studies confirmed this hypothesis. Based on such latency pattern, a scoring system was developed to quantify the neural differentiation of speech at the subcortical level and to facilitate its comparison with other behavioral measures of speech perception, etc. (Hornickel et al 2009). A higher score indicated that pairwise latency comparisons among phonemes earned high points by showing the aforementioned pattern in the expected direction, suggesting greater sub-cortical differentiation of stop consonants. As in the previous studies by Harkrider et al. (2005; 2006), spectral shaping moved older adults in the direction of the young adult norm. 
On the other hand; spectral-shaping reduced the stop consonant differentiation score in our younger adults. It is possible that shaping benefits accrued by older listeners are mediated at central auditory pathways, rather than at lower perceptual levels, as evident by shaping-related increases in N1 and P2 amplitudes in this population (Harkrider et al. 2005). Similar increases in amplitudes with shaping were not evident in younger adults (Harkrider et al. 2005). These greater responses may have been caused by compensatory mechanisms used by older adults to overcome perceptual deficits at the periphery. Older adults may have learned how to make use of all possible additional cues to understand speech, whereas, younger adults haven't learned how to employ such mechanisms due to often an intact perceptual conveyance of auditory signals. In both groups, the introduction of extra high frequency information may have resulted in interference with the expected latency pattern, a pattern that is based on the proportional high frequency components of the stimuli. Nevertheless, the experienced older adults were able to use the remapped spectral cues and showed sub-cortical improvements mediated by the efferent system with shaping, whereas younger adults did not.

Once again by observing our individual data (in Figure 4-5), spectral shaping allowed more older adults to exhibit the proposed latency progression for major peaks raising their total of 2 to 5 of 11 older adults (Figure 4-5). In contrast, just one younger adult showed the progressive pattern for the shaping condition. Minor peaks showed this progressive pattern in only one subject per each group once shaping was introduced. Individual data support the finding of shaping effects on the stop consonant differentiation score of major peaks; such that spectral shaping improved the score (of major peaks) in older adults, but reduced it in younger adults.

Furthermore, shaping increased the latencies of endpoint peaks. These peaks are assumed to reflect the very end of the formant transition period where the steady state period begins (Johnson et al. 2008). After the $40 \mathrm{~ms}$ formant transition, F2 remained constant throughout the steady state period. The steady state portion (i.e. $60 \mathrm{~ms}$ ) is longer than the transition portion (i.e. $40 \mathrm{~ms}$ ) of the stimuli used in this study and may have caused longer neural adaptation (Anderson et al. 2012). Schneider and Hamstra (1999) evaluated temporal resolution performances of older and younger adults with relatively normal hearing using gap detection tests. Thresholds of gap detection changed with marker durations, such that long marker durations adversely influenced gap detection performance. Their subjects were found to have elevated gap detection thresholds for long marker durations and vice versa. In comparison to the hypothesized firing patterns of neural units in primary auditory afferents, results of gap detection thresholds comply with a temporal window model that includes adaptation effects (Schneider and Hamstra 1999). According to this model, detecting marker durations depends on the extent of neural adaptation and the rapid recovery from it. As a consequence to such interpretation of such model, the longer duration (i.e. $60 \mathrm{~ms}$ ) of the steady state portion may have caused longer time course of neural adaptation than the shorter transition period (i.e. 40 $\mathrm{ms}$ ) in this present study. Additionally, spectral shaping may have caused more adaptation by introducing more high frequency information (Hellman, Miskiewicz, and Scharf 1997). As a result of neural adaptation, the latencies of endpoint peaks were more prolonged with shaping. 
Shaping reduced the latencies of major peaks for the $/ \mathrm{b} /$ phoneme. An explanation for this finding is that the F2 transition is rising for the /b/ phoneme; meanwhile it is falling for $/ \mathrm{d} /$ and $/ \mathrm{g} /$ phonemes. Due to spectral shaping, the $/ \mathrm{b} /$ phoneme is gaining more energy toward the steady state throughout the $40 \mathrm{~ms}$ F2 transition period. In general, increased stimulus intensities should enhance neural synchrony and shorten latencies. Therefore, latencies of major peaks of the /b/ phoneme were shortened by shaping.

Furthermore, shaping increased the amplitude of onset peaks for the /g/ phoneme. Spectral shaping increases gain for high frequency components. The phoneme $/ \mathrm{g} /$ had the highest F2 formant frequency among all stimuli. Thus, increased stimulus intensity enhanced neural synchrony and increased amplitude of onset peaks.

\section{Phoneme Effects}

Larger $/ \mathrm{b} /$ and /g/ categories and smaller /d/ category were found in both groups, suggesting that subjects were more sensitive to tokens at the extreme regions, i.e. $/ \mathrm{b} /$ and $\mathrm{g} / \mathrm{regions}$, than to tokens at the intermediate region of $/ \mathrm{da} /$. In other words, the $/ \mathrm{b} /$ and $\mathrm{g} / \mathrm{categories}$ were better defined than the $/ \mathrm{d} /$ category. This result is not surprising because $/ \mathrm{d} /$ can be confused by either $/ \mathrm{g} /$ or $/ \mathrm{d} /$, whereas $/ \mathrm{b} /$ is rarely labeled as $/ \mathrm{g} /$ or vice versa.

Amplitudes of onset peaks were larger for $/ \mathrm{d} /$ phonemes than for $/ \mathrm{b} /$ and $/ \mathrm{g} /$ phonemes. This finding makes sense because energy at onset is concentrated at low, mid, and high frequencies, for bilabials (i.e. /b/), velars (i.e. /g/), and alveolar (i.e. /d/) stop consonants, respectively (Liberman, Delattre, and Cooper 1952).

Amplitudes of major and minor peaks increased progressively from $/ \mathrm{b} /$ then to $/ \mathrm{d} /$ and finally to $/ \mathrm{g} /$. The stimulus with the highest F2 transition (i.e. $/ \mathrm{g} /$ ) elicited larger peak amplitudes than the stimulus with the mid-frequency F2 transition (i.e./d/), which elicited larger peak amplitudes than the stimulus with the lowest F2 transition (i.e. /b/). Due to such finding, it might be that amplitudes of minor and major peaks are reflecting the tonotopicity of the auditory brainstem. It is possible that both latency and amplitude encode frequency-related changes of phonemic contrasts in the voiced stop consonants. Increased speech-ABR amplitudes across phonemes may reflect a boost delivered by the auditory system to enhance phonemic contrasts, which could be an efferent interference for better tuning. Using cross-phaseogram analysis, Skoe et al. (2011) showed that phase delays of the speech- ABR can also contribute to the particular differentiation of the timevarying frequency transitions, different amplitudes and phase spectra among the three voiced stop consonants $/ \mathrm{b} /, / \mathrm{d} /$ and $/ \mathrm{g} /$. The phoneme of the highest frequency $/ \mathrm{g} / \mathrm{phase}$ leads the mid frequency phoneme $/ \mathrm{d} /$ and the low frequency phoneme $/ \mathrm{b} /$. Likewise, the $/ \mathrm{d} /$ phoneme phase leads the $/ \mathrm{b} /$. Therefore, a combination of latency, amplitude and phase analyses of the speech-ABR is necessary to track the neural representation of phonemic contrasts in stop consonants at the sub-cortical level. 


\section{Summary}

The first objective of this present investigation was to assess the influence of aging on the neural differentiation of stop consonants at the sub-cortical level. The second main objective was to determine whether or not spectral shaping modulates (i.e. improves) the sub-cortical responses in older adults (in case possible age-related adverse effects were obtained). The current study was also designed to determine whether possible age - related deficits at the sub-cortical level limit speech perception as assessed via categorical speech perception tasks. Finally, it was designed to determine whether potential neural improvements (possibly by shaping modulation) were associated with improved perception. By such study assessments, origins of mediation for phonemic processing were explored in normal-hearing aged listeners. To do so, three contrastive voiced stop consonant syllables (i.e. /b/, /d/, and /g/), with minimal differences on F2 formants, were used to evoke the speech-ABRs in our "normal-hearing" older and younger adults.

We hypothesized that if older adults with normal hearing exhibit abnormal stop consonant identification and if, at the same time, differences in the sub-cortical differentiation of contrastive stop consonants using speech-ABR were obtained between older adults with normal hearing and younger adults, then discriminating contrastive stop consonants in older adults with normal hearing would appear to be influenced by both the cortical, as evident by impaired cortical EPs in previous studies, and the sub-cortical levels, such that stop consonant discrimination might appear to be influenced by the interaction between bottom-up and top-down (i.e. corticofugal) processes in older adults.

On the other hand, we hypothesized that if the sub-cortical encoding of the contrastive stop consonants does not vary between normal-hearing older adults (proving the null hypothesis) then discrimination of stop consonants might appear to be mediated primarily by top-down processes. In this case, such finding may indicate a separation between sub-cortical and cortical contributions toward phonemic processing in normalhearing older adults, such that the cortical mediation is the sole and main influence on the phonemic perception in this population.

Our older adults did not show deficits in categorical perception using the behavioral speech tasks employed in this study. Our data revealed subtle electrophysiological differences between older adults and younger adult controls at the sub-cortical level. Individual data showed that the hypothesized latency pattern (i.e. a pattern that indicates robust sub-cortical differentiation among phonemes) for major peaks was less evident in older adults in comparison to younger adults, however, statistical group differences for these latencies were not achieved. Whether these differences between older and younger listeners are adequate to cause possible poor performance on the behavioral speech perception tasks or not cannot be teased out using the data of this current study. These subtle deficits at the sub-cortical level might have been over-ridden by cortical top-down compensation strategies in our high performing older adults. In other words, they might have adapted their speech perception abilities in response to these sub-cortical changes. Per decline-compensation hypothesis, it might be 
that our older adults counteracted age-related declines by a plastic reorganization of neuro-cognitive networks (Cabeza et al. 2002). It is possible that our older adults exerted more cognitive effort to overcome the sub-cortical deficits found in this current study or other possible cortical deficits. Without further cortical assessments to evaluate possible decline-compensation patterns, it is difficult to pinpoint the mechanisms (i.e. whether sub-cortical, cortical, or both levels) contributing toward the phonemic processing in normal-hearing older adults.

Differences in the behavioral area under the curve measure between phoneme pairs, and the speech-ABR differentiation scores for phoneme pairs (obtained for major peaks as well as minor peaks) showed no correlations suggesting one of the following: possible cortical interference to overcome sub-cortical impairments; sub-cortical impairments do not affect the behavioral identification of stop consonants; or sub-cortical impairments obtained in this study are so small to make any difference.

Taxing speech perception tasks at the phonemic level by presenting speech in challenging listening environments might help in evaluating the contribution of cognitive factors underlying stop consonant identification. If the performance of normal-hearing older adults on consonant perception task deviates from their original normal performance, or get worse by the introduction of noise or reverberation, then this might suggest top-down mediation and possible reduced inhibition of unwanted noise.

Many cognitive measurements, except for measures of processing speed, did not correlate with basic measures of speech perception (Martin \& Jerger 2005). Hence, the utilization of commonly used cognitive tests, such as tests for intelligence and memory span, may not be the best choice (Martin \& Jerger 2005) to assess cognitive levels in older adults. Therefore, accompanying taxed stop consonant speech perception tasks with appropriate cognitive tests such as the Paced Auditory Serial Addition Task (PASAT) test that assesses speed of processing might be more effective in exploring the mediation of phonemic processing in normal-hearing older adults.

The introduction of spectral shaping by enhancing the F2 transition improved the neural differentiation of phonemic contrasts at the brainstem level in our older adults, specifically; shaping increased their stop consonant differentiation score for major peaks. This finding is consistent with previous data reported by Harkrider et al. (2005; 2006), and suggests that the possible negative sub-cortical changes caused by aging are not irreparable (Harkrider et al. 2005). This also suggests that enhancing imperative information for stop consonant perception will improve neural responsiveness of aged but intact neurons. Thus, in case these sub-cortical impairments over-ride cortical compensation in otherwise less-performing normal-hearing older adults with reduced cognitive abilities, it is possible that their behavioral speech perception performance can be restored to "normal" by shaping. Behaviorally, older adults performed normally before and after the introduction of shaping, therefore, the extent to which shaping improved the speech perception of stop consonants in normal-hearing adults is unknown. 
Subjects with previously established difficulties in stop consonant perception might be tested sub-cortically to effectively verify shaping effects at the brainstem level. Shaping improved scores for some older adults, but not others. Future research should focus on differences within normal-hearing older adult groups to identify who benefits more from shaping and who might be adversely affected similarly to what happened in the case of younger adults.

According to our data, amplitudes of minor and major peaks seem to reflect the tonotopicity of the auditory brainstem. It is possible that frequency-related changes are being reflected in latency (e.g. Johnson et al 2008; Hornickel et al. 2009) and amplitude as well. Further research is required to investigate the precise effects of contrasting frequency and stimulus parameters on the sub-cortical neural encoding as assessed via the speech-ABR.

\section{Future Work}

It is imperative to extend the work of this current investigation to older adults with substantial hearing impairment (i.e. severe hearing loss). The findings of this present study provide a baseline from which we can evaluate the sub-cortical responses evoked by stop consonant stimuli in older adults with hearing loss. Speech-ABRs obtained from hearing-impaired older listeners can be compared to that of normal-hearing older adults in order to isolate age-related alterations from that of hearing loss at the sub-cortical level (Harkrider et al. 2005). The study by Harkrider et al. (2006) showed no improving effects of spectral shaping on the cortical neural responses obtained from hearing-impaired older adults, suggesting distorted neural firing, possibly caused by auditory deprivation, rather than a reduction in the responsiveness of intact neurons. Nevertheless, behavioral perception was improved in their hearing-impaired older population by shaping despite the persisting cortical neural deficits.

In contrast to the hearing-impaired results obtained in Harkrider et al. (2006), normal-hearing older adults (Harkrider et al. 2005) showed improvements in cortical responses as well as in behavioral performance in response to shaped stimuli. Such distinction between the behavioral and neural responses of normal-hearing and that of hearing-impaired older populations emphasizes the importance of further cortical as well as sub-cortical assessments. Hence, future work should focus on the effects of hearing loss and amplification on the sub-cortical neural representation of speech in older adults.

\section{Clinical Relevance}

By learning more about the interaction between the signal processing of hearing aids and the central auditory system we can improve our understanding of the sources underlying auditory rehabilitation (Billings, Tremblay, Souza \& Binns 2007). Auditory evoked potentials were reliably recorded and employed to assess amplification effects in literature (e.g. Tremblay, Billings, Friesen \& Souza 2006a; Billings et al. 2007). 
Specifically, recordings of cortical evoked potentials were obtained for auditory stimuli presented in sound field through loud speakers. Results showed that spectral and temporal alterations introduced by amplification seem to produce different response patterns compared to unaided recordings (e.g. Billings et al. 2007). Therefore, it is necessary to learn how amplification with its various settings affects neural patterns/representations of amplified speech sounds at all and different levels of the auditory pathway (Tremblay et al. 2006a).

It is possible that the neural representation of auditory stimuli can be enhanced by a simple change in hearing aids settings and/or by a certain rehabilitative strategy (Tremblay et al. 2006a). For example, if abnormal cortical evoked potentials accompany poor performance in speech perception, then providing slowly changing compression parameters might just improve the neural code. On the other hand, if normal neural encoding accompanies poor perception, then focusing on auditory training might maximize the use of neural encoding for a better perception (Tremblay et al. 2006a).

Previously, traditional-click ABR was used to electrophysiologically assess amplification benefits (e.g. Brown, Klein, \& Snyder, 1999; Beauchaine, Gorga, Reiland, et al., 1986); however such attempts weren't successful because the short click and tone pip stimuli used to elicit traditional ABRs did not effectively activate the circuitry of hearing aids (Tremblay et al. 2006a). Cortical evoked potentials uses speech syllables of long durations such as the $654.98-\mathrm{ms} / \mathrm{si} /$ and $/ \mathrm{ji} /$ syllables used in the study by Tremblay et al. (2006a).

So far, there is no empirical data with detailed procedural information in literature evaluating hearing aid fitting using speech-ABR. However, a study by Anderson and Kraus (in press) briefly mentioned the recording of speech-ABRs in sound field and the comparison of aided to unaided responses obtained using different hearing aid algorithms. The investigators' preliminary data showed amplitude differences between aided and unaided conditions, indicating the feasibility of using speech-ABR in the assessment and management of hearing impairment.

Due to the high fidelity of the speech-ABR in encoding speech signals and due to its high sensitivity in tracking stimulus changes, incorporating this measure into the verification of hearing aid performance seems a logical approach to study the effects of amplification on the brain. Speech-ABR may allow us to study auditory stimulation and deprivation at the central auditory system in older adults with hearing loss.

\section{Limitations of the Current Study}

One limitation for this present study is the small group of older adults $(n=11)$. Although most of the older adults recruited in this current investigation had normal hearing, some did not. Hence, hearing loss effects, although minimal in this present study, cannot be completely ruled out, and age effects cannot be purely assessed. Gender 
differences could not be explored as well; there were only 6 males in the younger adults group and 4 males in the older adults group.

Explicit measures of cognitive levels such as memory and attention were not controlled for in this current investigation. Insight into cognitive abilities could have helped in explaining the observed differences in the behavioral performance on speech perception tasks found in this present study. Recruiting subjects with previously established difficulties in speech perception could have contributed toward a better visualization and/or interpretation of the sub-cortical deficits and shaping effects in older adults.

The use of the same exemplar speech syllables for electrophysiological testing for all subjects might not have been ideal for each particular subject at the individual level. Stop consonant stimuli of this present study differed minimally from that of Johnson et al. (2008) and Hornickel et al. (2009) studies. Hence, comparisons on the sub-cortical differentiation of these stimuli among similar studies cannot be accurately achieved. However, the proposed latency patterns, based on the frequency-dependent hypothesis (Johnson et al. 2008), are expected to generalize across our stop consonant stimuli of /bd-g/. Stimuli in this present study were chosen to be similar to that used in Harkrider et al. $(2005 ; 2006)$ studies in order to uncover key information regarding spectral shaping. Another final limitation to this study is that individual differences between younger and older adults in the major peaks finding (as demonstrated in Figure 4-5) cannot be generalized beyond the subjects' sample.

\section{Conclusions}

1. Normal-hearing older adults had more robust categorical perception, or better defined categories than younger adult controls.

2. Possible cognitive involvement might contribute to the variability in speech perception seen in normal-hearing older adults.

3. Subtle sub-cortical differences were obtained between the two groups (older vs. younger adults).

4. In the control group, individual data showed robust progressive latency patterns for major peaks when compared to that of minor peaks.

5. In our subject sample, individual data showed sub-cortical stop consonant differentiation impairments in older adults as evident by missing progressive latency patterns for major peaks in this group (when compared to controls).

6. Spectral shaping improved the stop consonant differentiation score for major peaks in older adults, such that it moved older adults in the direction of the younger adults' norm. 


\section{LIST OF REFERENCES}

Abrams, D. A., \& Kraus, N. (2009). Auditory pathway representation of speech sounds in humans. In J. Katz, L. Hood, R. Burkard, \& L. Medwetsky (Eds.), Handbook of Clinical Audiology (pp. 611-626). Philadelphia: Lippincott, Williams, and Wilkins.

Agung, K., Purdy, S., McMahon, C., \& Newall, P. (2006). The use of cortical auditory evoked potentials to evaluate neural encoding of speech sounds in adults. Journal of the American Academy of Audiology, 17, 559-572.

Anderson, S., \& Kraus, N. (in press). The potential role of the cABR in assessment and management of hearing impairment. International Journal of Otolaryngology.

Anderson, S., Parbery-Clark, A., White-Schwoch, T., Kraus, N. (2012) Aging affects neural precision of speech encoding. Journal of Neuroscience, 32(41), $14156-$ 14164.

Anias, C. R., Lima, M. A. M. T., \& Kos, A. O. A. (2004). Evaluation of the influence of age in auditory brainstem response. Brazilian Journal of Otorhinolaryngology, 70, $84-89$.

Banai, K., \& Kraus, N. (2008). The dynamic brainstem: Implications for APD. In D. McFarland, \& A. Cacece (Eds.), Current controversies in central auditory processing disorder (pp. 269-289). San Diego, CA: Plural Publishing Inc.

Beauchaine, K. A., Gorga, M. P., Reiland, J. K., \& Larson, L. L. (1986). Application of ABRs to the hearing-aid selection process: preliminary data. Journal of Speech and Hearing Research, 29, 120-128.

Billings, C. J., Tremblay, K. L., Souza, P. E., \& Binns, M.A. (2007). Effects of hearing aid amplification and stimulus intensity on cortical auditory evoked potentials. Audiololgy and Neurotology, 12(4), $234-246$.

Blumstein, S. E., Baker, E., \& Goodglass, H. (1977a). Phonological factors in auditory comprehension in aphasia. Neuropsychologia, 15, 19-30.

Blumstein, S. E., Isaacs, E., \& Mertus, J. (1982). The role of the gross spectral shape as a perceptual cue to place articulation in initial stop consonants. Journal of the Acoustical Society of America, 72, 43-50.

Blumstein, S.E., \& Stevens, K.N. (1980). Perceptual invariance and onset spectra for stop consonants in different vowel environments. Journal of Acoustical Society of America, 67, 648662. 
Boothroyd, A. (1984). Auditory perception of speech contrasts by subjects with sensorineural hearing loss. Journal of Speech and Hearing Research, 27,134 144.

Boothroyd, A. (1991). Speech perception measures and their role in the evaluation of hearing aid performance. In J.A. Feigin \& P.G. Stelmachowicz (Eds.), Pediatric Amplification: Proceedings of the 1991 National Conference (pp 77-92). Omaha, NE: Boys Town National Research Hospital.

Brown, G., Klein, A. J., \& Snyder, K. (1999). Hearing-aid process tone pips: Electroacoustic and ABR characteristics. Journal of the American Academy of Audiology, 10, 190-197.

Cabeza, R., Anderson, N. D., Locantore, J. K., \& McIntosh, A. R. (2002). Aging gracefully: Compensatory brain activity in high-performing older adults. Neuroimage, 17, 1394-1402.

Darley, F.L., Aronson, A.E., \& Brown, J.R. (1969). Differential diagnostic patterns of dysarthria. Journal of Speech, Language, and Hearing Research, 12, 246-269.

Dehaene-Lambertz, G. (1997). Electrophysiological correlates of categorical phoneme perception in adults. NeuroReport, 8, 919-924.

Delgutte, B. (1980). Representation of speech-like sounds in the discharge patterns of auditory nerve fibers. Journal of Acoustical Society of America, 68, 843-857.

Dorman, M. F., Marton, K., \& Hannley, M. T. (1985). Phonetic identification by elderly normal and hearing-impaired listeners. Journal of the Acoustical Society of America, 77, 664-670.

Duffy, J. (1995). Motor Speech Disorders. St. Louis: Mosby.

Elangovan, S., \& Stuart, A. (2011). A cross-linguistic examination of cortical auditory evoked potentials for categorical voicing contrast. Neuroscience Letters, 490 (2), $140-144$.

Fitzgibbons, P. J., \& Gordon-Salant, S. (1996). Auditory temporal processing in elderly listeners. Journal of American Academy of Audiology, 7(3), 183- 189.

Frisina, D., \& Frisina, R. D. (1997). Speech recognition in noise and presbycusis: Relations to possible neural mechanisms. Hearing Research, 106, 95-104.

Galbraith, G. C., Arbagey, P. W., Branski, R., Comerci, N., \& Rector, P. M. (1995). Intelligible speech encoded in the human brain stem frequency following response. NeuroReport, 6, 2363-2367. 
Gibbs, S. (2004) Phonological awareness: An investigation into the developmental role of vocabulary and short-term memory. Educational Psychology, 24, 13-25.

Ginzel, A., Brahe Pedersen, C., Spliid, P. E., \& Andersen, E. (1982). The role of temporal factors in auditory perception of consonants and vowels. A study of different age groups. Scandinavian Audiology, 11, 93- 100.

Gordon-Salant, S. (1987). Age-related differences in speech recognition performance as a function of test format and paradigm. Ear and Hearing, 8(5), 270-276.

Gordon-Salant, S., Fitzgibbons, P. J. (1993). Temporal factors and speech recognition performance in young and elderly listeners. Journal of Speech and Hearing Research, 36(6), 1272-1285.

Hall, J. W. (2007). New handbook of auditory evoked responses. Boston: Pearson Education.

Harkrider, A. W., Plyler, P. N., \& Hedrick, M. S. (2005). Effects of age and spectral shaping on perception and neural representation of stop consonant stimuli. Clinical Neurophysiology, 116, 2153-2164.

Harkrider, A. W., Plyler, P. N., \& Hedrick, M. S. (2006). Effects of hearing loss and spectral shaping on perception and neural representation of stop consonant stimuli. Journal of the Acoustical Society of America, 120, 915-925.

Harkrider, A.W., Plyler, P.N., \& Hedrick, M.S. (2009). Effects of hearing loss and spectral shaping on identification and neural response patterns of stop-consonant stimuli in young adults. Ear and Hearing, 30, 31-42.

Harris, K.C., Dubno, J.R., Keren, N.I., Ahlstrom, J.B., \& Eckert, M.A. (2009). Speech recognition in younger and older adults: A dependency on low-level auditory cortex. Journal of Neuroscience, 29, 6078-6087.

Harris, K.C., Mills, J.H., \& Dubno, J.R. (2007). Electrophysiologic correlates of intensity discrimination in cortical evoked potentials of younger and older adults. Hearing Research, 228, 58-69.

Harris, K. C., Wilson, S., Eckert, M. A., \& Dubno, J.R. (2012). Human evoked cortical activity to silent gaps in noise: effects of age, attention, and cortical processing speed. Ear and Hearing, 33(3), 330-9.

Hedrick, M. S., Schulte, L., \& Jesteadt, W. (1995). Effect of relative and overall amplitude on perception of voiceless stop consonants by listeners with normal and impaired hearing. Journal of the Acoustical Society of America, 98, 1292-1303. 
Helfer, K., \& Huntley, R. (1991). Aging and consonant errors in reverberation and noise. Journal of the Acoustical Society of America, 90, 1786-1796.

Hellman, R., Miskiewicz, A., \& Scharf, B. (1997). Loudness adaptation and excitation patterns: Effects of frequency and level. The Journal of the Acoustical Society of America, 101, 21762185.

Helson, H. (1964). Adaptation-Level Theory. Harper \& Row. NY. NY.

Herholz, S.C., Lappe, C., \& Pantev, C. (2009). Looking for a pattern: An MEG study on the abstract mismatch negativity in musicians and nonmusicians. BMC Neuroscience, 10 (42), 1-10.

Hillyard, S. A., Hink, R. F., Schwent, V. L., \& Picton, T. W. (1973). Electrical signs of selective attention in the human brain. Science, 182 (4108), 177-180.

Hornickel, J., Knowles, E., \& Kraus, N. (2012). Reliability of the auditory brainstem responses to speech over year in school-age children: A Reply to Drs. McFarland and Cacace. Hearing Research, 287 (1-2), 3-5.

Hornickel, J., Skoe, E., Nicol, T., Zecker, S., \& Kraus, N. (2009). Subcortical differentiation of stop consonants relates to reading and speech-in-noise perception. Proceedings of the National Academy of Sciences of the United States of America, 106, 13022-13027.

Jacobson, G.P., Lombardi, D.M., Gibbens, N.D., Ahmad, B.K., \& Newman, C.W. (1992). The effects of stimulus frequency and recording site on the amplitude and latency of multichannel cortical auditory evoked potential (CAEP) component N1. Ear and Hearing, 13, 300-306.

Johnson, K. (1997b). The auditory/perceptual basis for speech segmentation. Ohio State University Working Papers in Linguistics, 50, 101-113.

Johnson, K. L., Nicol, T. G., \& Kraus, N. (2005). The brainstem response to speech: A biological marker of auditory processing. Ear and Hearing, 26, 424-34.

Johnson, K. L., Nicol, T., Zecker, S. G., Bradlow, A.R., Skoe, E., \& Kraus, N. (2008). Brainstem encoding of voiced consonant-vowel stop syllables. Clinical Neurophysiology, 119, 2623-2635.

Katz, J. (2002). Clinical Masking. In J. Katz (Ed.), Handbook Of Clinical Audiology (5 ${ }^{\text {th }}$ Ed., pp. 124-141). Philadelphia: Williams \& Wilkins.

King, C., Cunningham, J., Nicol, T., Warrier, C., \& Kraus, N., (2002). Deficits in auditory brainstem encoding of speech sounds in children with learning problems. Neuroscience Letters, 319, 111-115. 
Klatt, D. (1980). Software for a cascade/parallel formant synthesizer. Journal of the Acoustical Society of America, 67, 971-995.

Korczak, P., \& Stapells, D.R. (2010). Effects of various articulatory features of speech on cortical event-related potentials and behavioral measures of speech-sound processing. Ear and Hearing, 31, 491-504.

Kraus, N., \& Nicol, T. G. (2005). Brainstem origins for cortical "what" and "where" pathways in the auditory system. Trends in Neuroscience, 28, 176-181.

Liberman, A. M., Delattre, P., \& Cooper, F. S. (1952). The role of selected stimulusvariables in the perception of the unvoiced stop consonants. The American journal of psychology, 65 (4), 497-516.

Lister, J. J., Maxfield, N. D., Pitt, G. J., \& Gonzalez, V.B. (2011). Auditory evoked response to gaps in noise: Older adults. International Journal of Audiology, 50, 211-225.

Martin, J.S., \& Jerger, J.F. (2005). Some effects of aging on central auditory processing. Journal of Rehabilitative Research and Development, 42(4)(suppl 2), 25-44.

Maruthy, S., \& Mannarukrishnaiah, J. (2008): Effect of early onset otitis media on brainstem and cortical auditory processing. Behavioral and Brain Functions, 4 (17).

Mitchell, K. J., Raye, C. L., Johnson, M. K., \& Greene, E. J. (2006). An fMRI investigation of short-term source memory in young and older adults. NeuroImage, 30, 627-633.

Møller, A.R., \& Jannetta, P. (1985). Neural generators of the auditory brainstem response. In J. Jacobson (Ed.), The Auditory Brainstem Response (pp. 13-31). San Diego, CA: College-Hill Press.

Näätänen, R. (1992). Attention and brain function. Hillsdale, NJ: Lawrence Erlbaum Associates.

Näätänen, R. Lehtokoski, A., Lennest, M., Cheour, M., Huotilainen, M., livonen, A., et al. (1997). Language-specific phoneme representations revealed by electric and magnetic brain responses. Nature, 385, 432-434.

Näätänen, R., \& Picton, T. (1987). The N1 wave of the human electric and magnetic response to sound: A review and an analysis of component structure.

Psychophysiology, 24, 375-425. 
Ohde, R. N., \& Stevens, K. N. (1983). Effect of burst amplitude on the perception of stop consonant place of articulation. Journal of Acoustical Society of America, 74, 706-714.

Oku, T., \& Hasegewa, M. (1997). The influence of aging on auditory brainstem response and electrocochleography in the elderly. ORL Journal of Otorhinolaryngology and its Related Specialties, 59, 141-146.

Osterharnmel, P.A., Shallop, J.K., \& Terkildsen, K. (1985). The effect of sleep on the auditory brainstem response (ABR) and the middle latency response (MLR). Scandinavian Audiology, 14, 47-50.

Pichora-Fuller, M.K., \& Souza, P. (2003). Effects of aging on auditory processing of speech. International Journal of Audiology, 42 (Supp 2), S11-S16.

Picton, T.W., \& Hillyard, S. A. (1974). Human auditory evoked potentials. II. Effects of attention. Electroencephalography and Clinical Neurophysiology, 36(2), 191-199.

Picton, T.W., Stuss, D., Champagne, S., \& Nelson, R. (1984). The effects of age on human event-related potentials. Psychophysiology, 21, 312-94.

Plyler, P. N., \& Hedrick, M. S. (2002). The effects of stimulus presentation level on stop consonant identification in normal and hearing-impaired listeners. Journal of the American Academy of Audiology, 13, 154-159.

Potter, D. D., Pickles, C. D., Roberts, R. C., \& Rugg, M. D. (1992). The effects of scopolamine on event-related potentials in a continuous recognition memory task. Psychophysiology, 29, 29-37.

Ravizza, S. M. (2003). Dissociating the performance of cortical and subcortical patients in the perception and production of phonemes. Brain \& Cognition, 53(2), 301310 .

Raymer, A. M., Maher, L. M., Greenwald, M. L., Morris, M., Rothi, L. J. G., \& Heilman, K. M. (1990). Florida Semantics Battery. Unpublished test.

Russo, N., Nicol, T., Trommer, B., Zecker, S., \& Kraus, N. (2009). Brainstem transcription of speech is disrupted in children with autism spectrum disorders. Developmental Science, 12(4), 557-567.

Samra, S.K., Lilly, D.J., Rush, N.L., \& Kirsh, M.M. (1984). Fentanyl anesthesia and human brain-stem auditory evoked potentials. Anesthesiology, 261, 261-265.

Schneider, B.A., \& Hamstra, S.J. (1999). Gap detection thresholds as a function of tonal duration for older and younger adults. Journal of the Acoustical Society of America, 106, 371-380. 
Schnupp, J., Nelken, I., \& King, A. J. (2011). Auditory Neuroscience: Making Sense of Sound. Cambridge, MA: MIT Press.

Sharma, A., \& Dorman, M. (1999). Cortical Auditory evoked potential correlates of categorical perception of voice-onset time. Journal of the Acoustical Society of America, 106 (2), 1078-1083.

Singhal, A., Doerfling, P., \& Fowler, B. (2002). Effects of a dual task on the N100-P200 complex and the early and late Nd attention waveforms. Psychophysiology, 39, 236-245.

Skoe, E., \& Kraus, N. (2010). Auditory brain stem response to complex sounds: A tutorial. Ear and Hearing, 31, 302-324.

Skoe, E., Nicol, T., \& Kraus, N. (2011). Cross-phaseogram: Objective neural index of speech sound differentiation. Journal of Neuroscience Methods, 196, 308-317.

Song, J.H., Banai, K., Russo, N.M., \& Kraus, N. (2006). On the relationship between speech and nonspeech evoked auditory brainstem responses. Audiology and Neuro-Otolology, 11, 233-241.

Strouse, A., Ashmead, D. H., Ohde, R. N., \& Grantham, D.W. (1998). Temporal processing in the aging auditory system. Journal of the Acoustical Society of America, 104, 2385-2399.

Stuart, A., Phillips, D.P. (1996). Word recognition in continuous and interrupted broadband noise by young normal-hearing, older normal-hearing, and presbyacusic listeners. Ear and Hearing, 17(6), 478-489.

Surprenant, A.M. (2007). Effects of noise on identification and serial recall of nonsense syllables in older and younger adults. Aging, Neuropsychology, and Cognition, $14,126-141$.

Tremblay, K. M., Piskosz, M., \& Souza, P. (2002). Aging alters the neural representation of speech cues. NeuroReport, 13, 1865-1870.

Tremblay, K., Piskosz, M., \& Souza, P. (2003). Effects of age and age related hearing loss on the neural representation of speech cues. Clinical Neurophysiology, 114, 1332-1343.

Tremblay, K., \& Ross, B. (2007). Effects of age and age-related hearing loss on the brain. Journal of Communication Disorders, 40, 305-312.

Tremblay, K.L., Billings, C.J., Friesen, L.M., \& Souza, P.E. (2006a). Neural representation of amplified speech sounds. Ear and Hearing, 27, 93-103. 
Tun, P.A. (1998). Fast noisy speech: age differences in processing rapid speech with background noise. Psychology of Aging, 13, 424-434.

Vander Werff, K. R., \& Burns, K. S. (2011). Brain stem responses to speech in younger and older adults. Ear and Hearing, 32, 168-180.

Wall, L.G., Fox, R.A., Moenter, D., \& Dalebout, S.D. (1991). Effect of speech distinctions and age differences on auditory event-related potentials. Journal of the American Academy of Audiology, 2, 237-45.

Wang, X., \& Sachs, M.B. (1994). Neural encoding of single-formant stimuli in the cat. II. Responses of anteroventral cochlear nucleus units. Journal of Neurophysiology, 71, 59-78.

Willott, J.F., Parham, K., Hunter, K.P. (1991). Comparison of the auditory sensitivity of neurons in the cochlear nucleus and inferior colliculus of young and aging C57BL/6J and CBA/J mice. Hearing Research, 53, 78-94.

Wingfield, A., Tun, P. A., \& McCoy, S. L. (2005). Hearing loss in older adulthood: What it is and how it interacts with cognitive performance. Current Directions in Psychological Science, 14, 144-148.

Wong, P.C.M., Jin, J.X., Gunasekera, G.M., Abel, R., Lee, E.R., \& Dhar. S. (2009). Aging and Cortical Mechanisms of Speech Perception in Noise. Neuropsychologia, 47, 693-703. 


\section{VITA}

Dania Rishiq was born in Amman, Jordan in 1981. Between 1999 -2003 she studied communication sciences and disorders at the Applied Sciences University/ Amman, Jordan. She received an M.A. in Audiology from the University of Tennessee at Knoxville in 2008, and a Ph.D. in Speech and Hearing Sciences from the University of Tennessee Health Science Center in May 2013. Her doctoral research focused on the speech evoked physiologic responses and the temporal aspects of processing of sounds and speech through the auditory nervous system in disordered and aging populations. 